ITEP-TH-61/12

\title{
Laplacian growth in a channel and Hurwitz numbers
}

\author{
A. Zabrodin *
}

December 2012

\begin{abstract}
We study the integrable structure of the 2D Laplacian growth problem with zero surface tension in an infinite channel with periodic boundary conditions in the transverse direction. Similar to the Laplacian growth in radial geometry, this problem can be embedded into the 2D Toda lattice hierarchy in the zero dispersion limit. However, the relevant solution to the hierarchy is different. We characterize this solution by the string equations and construct the corresponding dispersionless tau-function. This tau-function is shown to coincide with the genus-zero part of the generating function for double Hurwitz numbers.
\end{abstract}

\section{Contents}

1 Introduction 2

2 Lax equations for the dToda hierarchy and associated growth processes 5

2.1 Dispersionless Lax equations . . . . . . . . . . . . . . . 5 5

2.2 General solution to the Lax equations . . . . . . . . . . . . . . 7

2.3 Contour dynamics . . . . . . . . . . . . . . . . . . . . 9

2.4 The dispersionless tau-function . . . . . . . . . . . . . 11

2.5 Example: Laplacian growth in radial geometry . . . . . . . . . . . . 12

*Institute of Biochemical Physics, 4 Kosygina st., Moscow 119334, Russia; ITEP, 25 B.Cheremushkinskaya, Moscow 117218, Russia and National Research University Higher School of Economics, 20 Myasnitskaya Ulitsa, Moscow 101000, Russia, e-mail: zabrodin@itep.ru 
3 Laplacian growth in channel geometry 13

3.1 The moving boundary value problem . . . . . . . . . . 13

3.2 A growth problem in the auxiliary physical plane ......... 14

3.3 The tau-function . . . . . . . . . . . . . . . . 16

3.4 Example: the case of non-zero $t_{0}, t_{1}$ (trochoid) $\ldots \ldots \ldots \ldots$

4 The LG tau-function as the generating function for genus zero double Hurwitz numbers

5 Conclusion 25

Acknowledgments 25

References $\quad 25$

\section{Introduction}

Growth problems of Laplacian type such as Hele-Shaw viscous flows refer to dynamics of a moving front (an interface) between two distinct phases driven by a harmonic scalar field. These essentially nonlinear and non-local problems attract much attention for quite a long time [1]. The Laplacian growth problem appears in different physical and mathematical contexts and has important practical applications. The most known ones are filtration processes in porous media, viscous fingering in the Hele-Shaw cell, electrodeposition and solidification in undercooled liquids. The problem in 2D is the most studied one. For reviews see [2, 3, 4. To be definite, we shall speak about the dynamics of an interface between two incompressible fluids with very different viscosities. In practice the 2D geometry is realized in the Hele-Shaw cell - a narrow gap between two parallel plates (Fig. 1).

Remarkably, 2D Laplacian growth (LG) with vanishing surface tension possesses a hidden integrable structure which, for the problem in the radial geometry, i.e., in the plane with a point-like source or sink, was revealed in [5] and further studied in [8-[14]. Since evolution of planar simply-connected domains is most naturally described by timedependent conformal maps, there is no surprise that this structure is actually immanent for general conformal maps and classical boundary value problems. Specifically, it was shown in [5, 6] that evolution of conformal maps is governed by an integrable hierarchy of nonlinear partial differential equations which is a zero dispersion version [15, 16] of the 2D Toda hierarchy [17]. We call it the dispersionless Toda (dToda) hierarchy. The times of the hierarchy are harmonic moments of the evolving domain and the Lax function is identified with the conformal map. In fact the dispersionless Lax equations for it can be derived in the framework of the classical theory of conformal maps depending on parameters.

It is also remarkable that the integrable structure unites the LG problem with important areas of mathematics and theoretical physics such as inverse potential problem, 


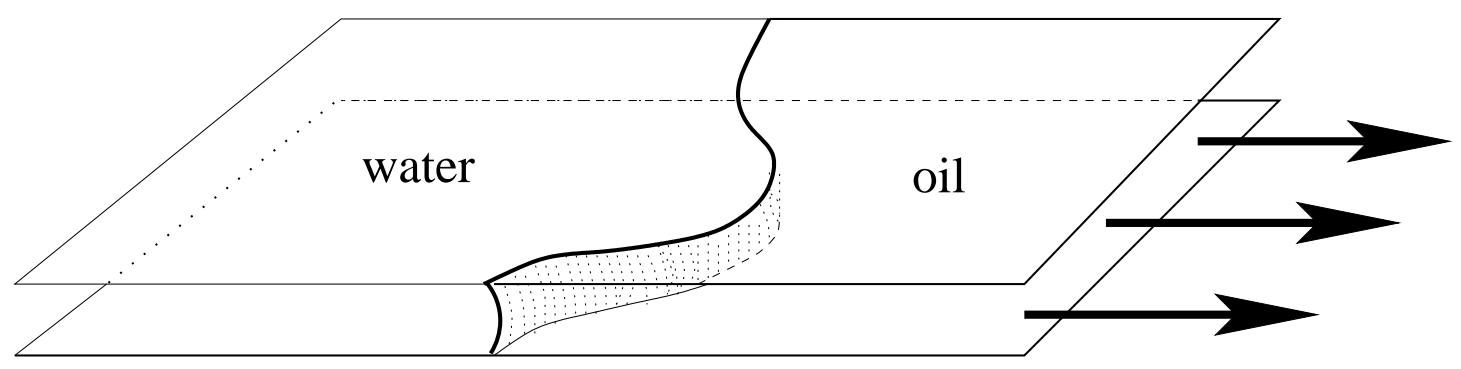

Figure 1: The Hele-Shaw cell.

quadrature domains, random matrices, theory of solitons and $c=1$ string theory. Some of these links are reviewed in [18]. We are going to add a new item to the list.

One aim of this work is to make explicit the integrable structure of the LG problem in a different geometry - namely in an infinite channel with periodic boundary conditions in the transverse direction (an infinite cylinder). In this version, the problem is also known as the Saffman-Taylor problem. We will show that the evolution is governed by the same dispersionless Lax equations but the specific solution of the dToda hierarchy is substantially different from the one relevant to the LG growth process in the plane.

Another aim is to show that the solution to the LG on a cylinder has an intriguing combinatorial and algebro-geometrical meaning. Namely, it appears to be closely related to the enumerative geometry of ramified coverings of the Riemann sphere. The corresponding tau-function turns out to be the dispersionless limit of the tau-function for Hurwitz numbers which is the generating function for numbers counting ramified coverings of $\mathbb{C P}^{1}$ of a certain ramification type (see [19] for a review of the Hurwitz problem and related topics). The combinatorial theory of ramified coverings of $\mathbb{C P}^{1}$ was linked to integrable systems in [20, 21]. In particular, the generating function for double Hurwitz numbers was shown to be a special solution (tau-function) of the 2D Toda lattice hierarchy in [21]. The integrability of Hurwitz partition functions and their relation to matrix models is now actively investigated (see e.g. [22]-[27]).

In the rest part of the introduction we outline the contents of the paper.

The LG problem on the surface of a cylinder. Consider an infinite cylinder of radius $R$ obtained from the physical $(X, Y)$ plane by identifying the points $(X, Y+2 \pi m R)$ for all $m \in \mathbb{Z}$. As usual, we will use the complex coordinates $Z=X+i Y, \bar{Z}=X-i Y$. Let $\Gamma$ be a closed non-intersecting contour on the cylinder equivalent to the non-trivial cycle. It divides the cylinder into two infinite pieces, $D_{-}$and $D_{+},-$to the left and to the right of $\Gamma$ respectively. Without loss of generality we assume that the section $X=0$ lies entirely in $D_{-}$(Fig. 2). Let the viscous fluid (oil) in $D_{+}$be sucked by a pump on the right infinity, with the non-viscous fluid (water) coming to $\mathrm{D}_{-}$from the left infinity, then the interface $\Gamma$ moves to the right, with the normal velocity $V_{n}=V_{n}(Z)$ at any point $Z \in \Gamma$ being given by

$$
V_{n}(Z)=-\partial_{n} \Phi(Z) .
$$

The potential function $\Phi$ is proportional to the pressure in the viscous fluid. It is a harmonic function in $D_{-}$equal to 0 on $\Gamma$ (zero surface tension) with the asymptotic behaviour $\Phi \sim-\frac{1}{2} X$ as $X \rightarrow+\infty$. The velocity field in the viscous fluid is given by the Darcy law $\vec{V}=-\vec{\nabla} \Phi$. 


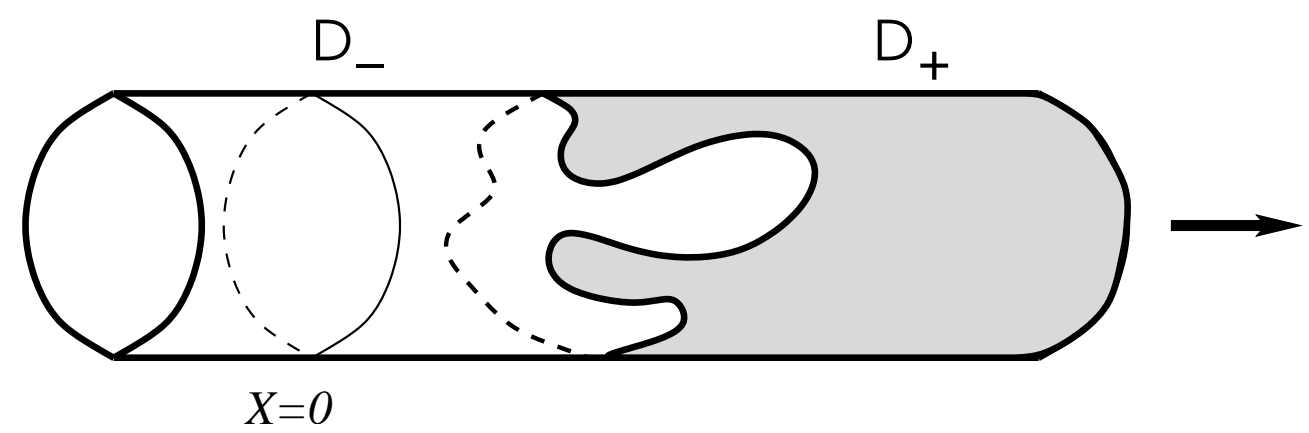

Figure 2: Laplacian growth in a channel with periodic boundary conditions (a cylinder).

The simply-connected case (a single interface) allows for an effective application of the conformal mapping technique (see, e.g., [2, 28)). In the complex coordinates $Z, \bar{Z}$ one may describe the growth process in terms of a time dependent conformal map $Z(W, t)$ from a fixed domain of a simple form in the "mathematical" $W$-plane, say the half-strip, onto the evolving oil domain in the "physical" $Z$-plane. The interface itself is the image of the segment $[0,2 \pi i]$ of the imaginary axis. The Hele-Shaw dynamics is then translated to a nonlinear partial differential equation for the function $Z(W, t)$, referred to as the Laplacian growth equation [29]:

$$
2 \mathcal{I} m\left(\partial_{\sigma} Z(i \sigma) \partial_{t} \overline{Z(i \sigma)}\right)=R, \quad \sigma \in[0,2 \pi] .
$$

The harmonic moments of the oil domain $\mathrm{D}_{+}$(Richardson's moments),

$$
t_{k}=-\frac{1}{\pi k R} \iint_{\mathrm{D}_{+}} e^{-k Z / R} d X d Y, \quad k \geq 1,
$$

are known to be constants of motion for the LG process [30]. The complimentary set of moments,

$$
v_{k}=\frac{1}{\pi R} \iint_{\mathrm{D}_{-}} e^{k Z / R} d X d Y, \quad k \geq 1,
$$

are time-dependent quantities.

The dToda hierarchy and the dispersionless tau-function. A direct derivation of the dToda hierarchy along the lines of [5, 6] is possible for the LG on a cylinder but we will follow a more formal approach suggested in [14] and map our problem to a contour dynamics in the radial geometry taking place in an "auxiliary physical plane" (the $z$-plane) with a non-uniform density. Contrary to the LG in the plane, the Lax function of the dToda hierarchy for the interface dynamics on the cylinder is not the conformal map $Z(W)$ itself but the exponential function $z=e^{Z(W) / R}$. The hierarchical times are Richardson's moments $t_{k}$. The LG equation (1.2) plays the role of the "string equation" which uniquely characterizes the solution to the whole hierarchy. We construct the dispersionless tau-function for this solution,

$$
F_{0}\left(R ; t_{0},\left\{t_{k}\right\}_{k \geq 1},\left\{\bar{t}_{k}\right\}_{k \geq 1}\right),
$$

which is a function of the harmonic moments $t_{k}$, the variable $t_{0}$ related to the area of the growing domain and depends on $R$ as a parameter. The function $F_{0}$ obeys the 
dispersionless Hirota equations for the dToda hierarchy

$$
\begin{gathered}
\left(z_{1}-z_{2}\right) e^{D\left(z_{1}\right) D\left(z_{2}\right) F_{0}}=z_{1} e^{-\partial_{t_{0}} D\left(z_{1}\right) F_{0}}-z_{2} e^{-\partial_{t_{0}} D\left(z_{2}\right) F_{0}}, \\
z_{1} \bar{z}_{2}\left(1-e^{-D\left(z_{1}\right) \bar{D}\left(\bar{z}_{2}\right) F_{0}}\right)=e^{\partial_{t_{0}}\left(\partial_{t_{0}}+D\left(z_{1}\right)+\bar{D}\left(\bar{z}_{2}\right)\right) F_{0}},
\end{gathered}
$$

where $D(z)=\sum_{k \geq 1} \frac{z^{-k}}{k} \partial_{t_{k}}, \bar{D}(\bar{z})=\sum_{k \geq 1} \frac{\bar{z}^{-k}}{k} \partial_{\bar{t}_{k}}$. It contains all information about the LG process and the conformal maps in the sense that it allows one to find the complimentary moments $v_{k}$ and the (inverse) conformal map $W(Z)$ by the formulas

$$
v_{k}=\frac{\partial F_{0}}{\partial t_{k}}, \quad W(Z)=\frac{Z}{R}-\frac{1}{2} \frac{\partial^{2} F_{0}}{\partial t_{0}^{2}}-\sum_{k \geq 1} \frac{e^{-k Z / R}}{k} \frac{\partial^{2} F_{0}}{\partial t_{0} \partial t_{k}} .
$$

The dToda hierarchy is an example of the universal Whitham hierarchy introduced in [15]. It is a multi-dimensional extension of the hierarchies of hydrodynamic type [31. The solutions can be parametrized [16] by canonical transformations in a two-dimensional phase space in such a way that any solution corresponds to a canonical pair of functions (the "twistor data"). In fact, this is equivalent to the characterization of the solutions via string equations.

The connection with Hurwitz numbers. We also show that the function $F_{0}$ is closely connected with the genus zero part of the generating function for the double Hurwitz numbers that count connected coverings of the sphere. The precise conneciton is as follows:

$$
F_{0}=\frac{t_{0}^{3}}{6 R}+\sum_{d \geq 1} e^{d t_{0} / R} \sum_{|\mu|=|\bar{\mu}|=d} \frac{R^{2-\ell(\mu)-\ell(\bar{\mu})}}{(\ell(\mu)+\ell(\bar{\mu})-2) !} H_{d, \ell(\mu)+\ell(\bar{\mu})-2}(\mu, \bar{\mu}) \prod_{i=1}^{\ell(\mu)} \mu_{i} t_{\mu_{i}} \prod_{i=1}^{\ell(\bar{\mu})} \bar{\mu}_{i} \bar{t}_{\bar{\mu}_{i}} .
$$

Here $\mu, \bar{\mu}$ are partitions of $d=|\mu|=|\bar{\mu}|$ into $\ell(\mu)$ parts $\mu_{1} \geq \mu_{2} \geq \ldots \geq \mu_{\ell(\mu)}>0$ (respectively, into $\ell(\bar{\mu})$ parts $\bar{\mu}_{1} \geq \bar{\mu}_{2} \geq \ldots \geq \bar{\mu}_{\ell(\bar{\mu})}>0$ ), $H_{d, l}(\mu, \bar{\mu})$ is the properly weighted number of topologically non-equivalent coverings $f: \mathbb{C P}^{1} \longrightarrow \mathbb{C P}^{1}$ of degree $d$ having ramification points at 0 and $\infty$ of the types $\mu$ and $\bar{\mu}$ respectively and $l=$ $\ell(\mu)+\ell(\bar{\mu})-2$ simple ramification points. The numbers $H_{d, l}(\mu, \bar{\mu})$ are called the double Hurwitz numbers [21]. In the series (1.7) only Hurwitz numbers corresponding to the genus-zero coverings enter. We see that they are basically the Taylor series coefficients of the dispersionless tau-function (1.3).

\section{Lax equations for the dToda hierarchy and associ- ated growth processes}

In this section we review some results of [5]-[14] in the form convenient for our purposes.

\subsection{Dispersionless Lax equations}

We start with the Lax equations for the dToda hierarchy with certain reality conditions imposed. The main object is the Lax function $z(w)$ represented as a Laurent series of 
the form

$$
z(w)=r w+a_{0}+\frac{a_{1}}{w}+\frac{a_{2}}{w^{2}}+\ldots
$$

The leading coefficient $r$ is assumed to be real while all other coefficients $a_{i}$ are in general complex numbers. All the coefficients depend on deformation parameters (or "times") $t_{0}$ (a real number) and $t_{1}, t_{2}, t_{3}, \ldots$ (complex numbers) in accordance with the Lax equations in the Sato form

$$
\frac{\partial z(w)}{\partial t_{k}}=\left\{A_{k}(w), z(w)\right\}, \quad \frac{\partial z(w)}{\partial \bar{t}_{k}}=-\left\{\bar{A}_{k}\left(w^{-1}\right), z(w)\right\}
$$

where for any two functions of $w, t_{0}$ the Poisson bracket is

$$
\{f, g\}:=\frac{\partial f}{\partial \log w} \frac{\partial g}{\partial t_{0}}-\frac{\partial f}{\partial t_{0}} \frac{\partial g}{\partial \log w} .
$$

Here and below the bar denotes the complex conjugation and $\bar{f}(w)=\overline{f(\bar{w})}$. The reality condition implies that the second half of the Lax equations (with $\bar{t}_{k}$-derivatives) is obtained from the first one by complex conjugation provided $w$ belongs to the unit circle. The generators of the flows are

$$
A_{k}(w)=\left(z^{k}(w)\right)_{+}, \quad A_{0}(w)=\log w
$$

For the dToda hierarchy, the $(\ldots)_{+}$-operation is

$$
\left(z^{k}(w)\right)_{+}:=\left(z^{k}(w)\right)_{>0}+\frac{1}{2}\left(z^{k}(w)\right)_{0} .
$$

Hereafter, $(\ldots)_{S}$ means taking the terms of the Laurent series with degrees belonging to the subset $S \in \mathbb{Z}$ (in particular, $(\ldots)_{0}$ is the free term). Note that at $k=0$ equations (2.2) become tautological identities. The second Lax function of the dToda hierarchy is $\bar{z}\left(w^{-1}\right)$. The reality conditions (i.e. the requirement that its coefficients are complex conjugate to those of the $z(w))$ imply that it obeys the same Lax equations.

By purely algebraic manipulations, one can show [15, 16] that the Lax equations (2.2) with $A_{k}$ given by (2.4) are equivalent to

$$
\begin{aligned}
& \partial_{t_{j}} A_{k}(w)-\partial_{t_{k}} A_{j}(w)+\left\{A_{k}(w), A_{j}(w)\right\}=0, \\
& \partial_{t_{j}} \bar{A}_{k}\left(w^{-1}\right)+\partial_{\bar{t}_{k}} A_{j}(w)+\left\{\bar{A}_{k}\left(w^{-1}\right), A_{j}(w)\right\}=0,
\end{aligned}
$$

which is a dispersionless version of the "zero curvature" representation. In their turn, equations (2.5) imply that the Lax equations (2.2) are compatible with each other, i.e., $\partial_{t_{n}}\left(\partial_{t_{m}} z(w)\right)=\partial_{t_{m}}\left(\partial_{t_{n}} z(w)\right)$ and $\partial_{\bar{t}_{n}}\left(\partial_{t_{m}} z(w)\right)=\partial_{t_{m}}\left(\partial_{\bar{t}_{n}} z(w)\right)$ for all $n, m$. This means that the vector fields $\partial_{t_{k}}, \partial_{\bar{t}_{n}}$ commute and these symbols can be understood as partial derivatives.

Let $w(z)$ be the inverse function to the Lax function $z(w)$. In terms of the inverse function, the evolution equations (2.2) acquire a simpler form:

$$
\frac{\partial \log w(z)}{\partial t_{k}}=\frac{\partial A_{k}}{\partial t_{0}}, \quad \frac{\partial \log w(z)}{\partial \bar{t}_{k}}=-\frac{\partial \bar{A}_{k}}{\partial t_{0}} .
$$


Here $A_{k}=A_{k}(w(z)), \bar{A}_{k}=\bar{A}_{k}(1 / w(z))$ are regarded as functions of $z$, and the derivatives are taken at fixed $z$. Treating $A_{k}$ 's as functions of $z$, one can rewrite equations (2.5) in the form similar to (2.6):

$$
\frac{\partial A_{j}}{\partial t_{k}}=\frac{\partial A_{k}}{\partial t_{j}}, \quad \frac{\partial A_{j}}{\partial \bar{t}_{k}}=-\frac{\partial \bar{A}_{k}}{\partial t_{j}} .
$$

Note that at $j=0$ this system coincides with (2.6).

It follows from the construction of the $A_{k}$ 's that the expansion of $A_{k}(w(z))$ in a Laurent series in $z$ is of the form $A_{k}=z^{k}+O(1)$. More precisely, these Laurent series are

$$
\begin{gathered}
A_{0}(w(z))=\log w(z)=\log z-\frac{1}{2} \partial_{t_{0}} v_{0}-\sum_{k \geq 1} \frac{\partial_{t_{0}} v_{k}}{k} z^{-k}, \\
A_{j}(w(z))=z^{j}-\frac{1}{2} \partial_{t_{j}} v_{0}-\sum_{k \geq 1} \frac{\partial_{t_{j}} v_{k}}{k} z^{-k}, \quad j \geq 1, \\
\bar{A}_{j}\left(w^{-1}(z)\right)=\frac{1}{2} \partial_{\bar{t}_{j}} v_{0}+\sum_{k \geq 1} \frac{\partial_{\bar{t}_{j}} v_{k}}{k} z^{-k}, \quad j \geq 1,
\end{gathered}
$$

where $v_{k}$ are functions of the times such that $\partial_{t_{j}} v_{k}=\partial_{t_{k}} v_{j}, \partial_{t_{j}} \bar{v}_{k}=\partial_{\bar{t}_{k}} v_{j}$.

We are especially interested in the class of solutions such that $z(w)$, for all $t_{k}$ in an open set of the space of parameters, is a univalent function in a neighborhood of infinity including the exterior of the unit circle. This means that $z\left(w_{1}\right)=z\left(w_{2}\right)$ if and only if $w_{1}=w_{2}$. From now on, we assume that $z(w)$ belongs to this class. In this case $z(w)$ is a conformal map from the exterior of the unit circle to a domain in the complex plane containing infinity while $\bar{z}\left(w^{-1}\right)$ is a conformal map from the interior of the unit circle to the complex conjugate domain. For technical reasons it is convenient to assume that the origin of the $z$-plane lies outside this domain.

\subsection{General solution to the Lax equations}

A general solution to the system of differential equations (2.2) is available in an implicit form [16]. To present it, we need an extended version of the Lax formalism.

By the definition of the Poisson bracket, $\log w$ and $t_{0}$ form a canonical pair:

$$
\left\{\log w, t_{0}\right\}=1 \text {. }
$$

The evolution according to the Lax equations can be regarded as a $t_{k}$-dependent canonical transformation from the pair $\left(\log w, t_{0}\right)$ to another canonical pair whose first member is $\log z(w)$. It is quite natural to introduce the second member which we denote by $M$. Depending on the situation, we treat it either as a function of $z$ and $t_{0}$ or as a function of $w$ and $t_{0}$ through the composition $M=M\left(z\left(w, t_{0}\right), t_{0}\right)$ (it also depends on the deformation parameters $\left.t_{k}\right)$. To find what is $M$, we note that the condition $\{\log z, M\}=1$ can be identically rewritten as $\partial_{t_{0}} M(z)=z \partial_{z} \log w\left(z, t_{0}\right)$. It determines $M$ up to a term depending only on $z$. The latter can be fixed by the requirement that $M$ obeys the same Lax equations (2.2). To wit, equation $\partial_{t_{k}} M=\left\{A_{k}, M\right\}$ (where the derivatives are taken at constant $w$ ) is equivalent to

$$
\partial_{t_{k}} M(z)=w \partial_{w} A_{k} \partial_{t_{0}} M(z)=z \partial_{z} A_{k}
$$


Taking into account (2.8), (2.9), we can write

$$
M(z)=\sum_{k \geq 1} k t_{k} z^{k}+t_{0}+\sum_{k \geq 1} v_{k} z^{-k}
$$

This formal Laurent series represents an analytic function if the domains of analyticity for the functions represented by the series $\sum_{k>1} k t_{k} z^{k}$ and $\sum_{k>1} v_{k} z^{-k}$ overlap. The function $M$ is the dispersionless ("quasiclassical") limit of the Orlov-Shulman operator [32]. Its geometric meaning depends on a particular solution. In a similar way, one can construct the conjugate Orlov-Shulman function, $\bar{M}(\bar{z})$, such that the transformation $\left(\log w, t_{0}\right) \rightarrow$ $\left(\log \bar{z}^{-1}\left(w^{-1}\right), \bar{M}\left(\bar{z}\left(w^{-1}\right)\right)\right.$ is canonical and $\bar{M}$ obeys the same Lax equations. The Lax equations imply that the composition of the canonical transformations

$$
(\log z, M) \rightarrow\left(\log w, t_{0}\right) \rightarrow\left(\log \bar{z}^{-1}, \bar{M}\right)
$$

does not depend on $t_{k}$, i.e., it is an integral of motion. Moreover, any $t_{k}$-independent canonical transformation $(\log z, M) \rightarrow\left(\log \bar{z}^{-1}, \bar{M}\right)$ between the Laurent series of the form prescribed above generates a solution to the dToda hierarchy. See [16] for the detailed proof.

More precisely, let $\left(\log f\left(w, t_{0}\right), g\left(w, t_{0}\right)\right)$ be a canonical pair: $\{\log f, g\}=1$. Suppose that the functions $z, \bar{z}, M, \bar{M}$ are represented by Laurent series of the form given above and are connected by the functional relations

$$
1 / \bar{z}\left(w^{-1}\right)=f(z(w), M(z(w))), \quad \bar{M}\left(\bar{z}\left(w^{-1}\right)\right)=g(z(w), M(z(w))) .
$$

Then the function $z(w)$ obeys all the Lax equations and its coefficients (as functions of $t_{k}$ 's) obey the dToda hierarchy. Conversely, any solution of the dToda hierarchy admits a representation of this form with some $(f, g)$-pair [16].

This construction can be made more explicit by introducing the generating function of the canonical transformation $\left(\log w, t_{0}\right) \rightarrow(\log f, g)$. An important class of solutions corresponds to the canonical transformations $(\log z, M) \rightarrow\left(\log \bar{z}^{-1}, \bar{M}\right)$ defined by means of a generating function $U(z, \bar{z})$ as follows:

$$
M=z \partial_{z} U(z, \bar{z}), \quad \bar{M}=\bar{z} \partial_{\bar{z}} U(z, \bar{z}) .
$$

Here $U(z, \bar{z})$ is an arbitrary differentiable real-valued function of $z, \bar{z}$. This form of the canonical transformation implies that the functions $z(w)$ and $\bar{z}\left(w^{-1}\right)$ are algebraically independent. These are solutions of generic type and we call them non-degenerate. They describe conformal maps of $2 \mathrm{D}$ domains with smooth boundaries. For non-degenerate solutions the "string equation"

$$
\left\{z(w), \bar{z}\left(w^{-1}\right)\right\}=\frac{1}{U_{z \bar{z}}\left(z(w), \bar{z}\left(w^{-1}\right)\right)}
$$

where $U_{z \bar{z}}(z, \bar{z}) \equiv \partial_{z} \partial_{\bar{z}} U(z, \bar{z})$ holds true. It is obtained by plugging $M$ from (2.13) into the canonical relation $\{z, M\}=z$. 

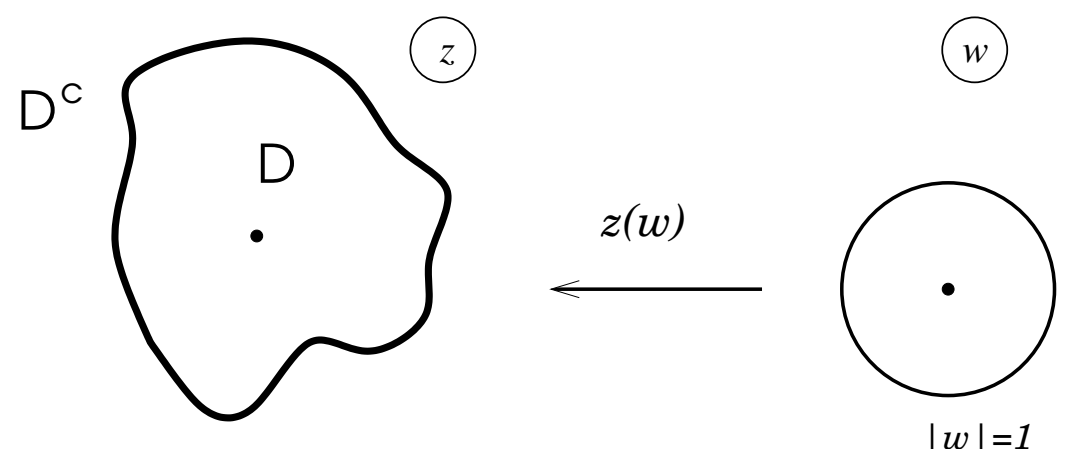

Figure 3: The Lax contour.

\subsection{Contour dynamics}

Lax equations (2.2) can be treated as equations of a contour dynamics. The contour is the image of the unit circle, i.e., $z\left(e^{i \theta}\right), 0 \leq \theta \leq 2 \pi$ (Fig. 3). We call it the Lax contour and denote it by $\gamma$. It depends on the deformation parameters. We assume that $\gamma$ is a non-self-intersecting curve encircling the origin. The function $z(w)$ provides a timedependent conformal map from the exterior of the unit circle onto the exterior of the Lax contour.

To derive equations of motion for the Lax contour, we need a general kinematic relation. Let $(x(\sigma, t), y(\sigma, t))$ be any parameterizations of a moving closed contour in the plane, then the normal velocity of the contour points is $V_{n}=\frac{d \sigma}{d l}\left(\partial_{\sigma} y \partial_{t} x-\partial_{\sigma} x \partial_{t} y\right)$, or, in the complex notation,

$$
V_{n}=\frac{d \sigma}{2 d l}\left(\partial_{\sigma} z \partial_{t} \bar{z}-\partial_{\sigma} \bar{z} \partial_{t} z\right),
$$

where $d l=\sqrt{(d x)^{2}+(d y)^{2}}$ is the line element. The normal velocity $V_{n}$ is positive if it is directed to the exterior of the contour.

Applying this formula to the Lax contour $z\left(e^{i \theta}\right)$ with the specific parameterization $\sigma=\theta$ and $t=t_{0}$ with all other $t_{k}$ 's fixed, we get the normal velocity of the Lax contour $\gamma$ at the points $z(w),|w|=1$ :

$$
V_{n}=\frac{\left\{z(w), \bar{z}\left(w^{-1}\right)\right\}}{2\left|z^{\prime}(w)\right|} .
$$

Here $z^{\prime}(w)=\partial_{w} z(w)$ and the Poisson bracket in the numerator is given by (2.3). Eq. (2.16) together with the string equation (2.14) states that the normal velocity of the Lax contour at the point $z \in \gamma$ is equal to

$$
V_{n}(z)=\frac{\left|w^{\prime}(z)\right|}{2 \partial_{z} \partial_{\bar{z}} U(z, \bar{z})}, \quad z \in \gamma .
$$

Eqs. (2.11), (2.13) allow us to express the deformation parameters through the geometry of the moving contour:

$$
t_{k}=\frac{1}{2 \pi i k} \oint_{|w|=1} z^{-k-1}(w) M(z(w)) d z(w)=\frac{1}{2 \pi i k} \oint_{\gamma} z^{-k} \partial_{z} U d z, \quad k \geq 1,
$$




$$
t_{0}=\frac{1}{2 \pi i} \oint_{|w|=1} M(z(w)) d \log z(w)=\frac{1}{2 \pi i} \oint_{\gamma} \partial_{z} U d z .
$$

We stress that $t_{1}, t_{2}, \ldots$ are kept constant, so they are integrals of motion for the contour dynamics (2.17). Let $\mathrm{D}$ be the compact domain bounded by the Lax contour and $\mathrm{D}^{\mathrm{c}}=$ $\mathbb{C} \backslash D$ its complement, then Green's formula implies that

$$
\begin{aligned}
t_{k} & =-\frac{1}{4 \pi k} \iint_{\mathrm{D}^{c}} z^{-k} \Delta U d^{2} z, \quad k \geq 1 \\
t_{0} & =\frac{1}{4 \pi} \iint_{\mathrm{D}} \Delta U d^{2} z
\end{aligned}
$$

where $\Delta=4 \partial_{z} \partial_{\bar{z}}$ is the Laplace operator and $d^{2} z \equiv d x d y$. According to our assumption, the domain $\mathrm{D}$ contains the origin, so the integrals (2.20) with positive $k$ are well-defined (for small $k$ a regularization at infinity is required). These formulas show that the $t_{k}$ 's are harmonic moments with the density function $\Delta U$. The double integral representation of $t_{0}$ implies that the density function is integrable everywhere in $\mathrm{D}$. If $\Delta U$ is singular at some point (say, at $z=0$ ), then one still may give sense to this double integral by introducing a cut-off (see the next section). The coefficients $v_{k}$ in (2.11) have similar integral representations:

$$
v_{k}=\frac{1}{2 \pi i} \oint_{|w|=1} z^{k-1}(w) M(z(w)) d z(w)=\frac{1}{2 \pi i} \oint_{\gamma} z^{k} \partial_{z} U d z=\frac{1}{4 \pi} \iint_{\mathrm{D}} z^{k} \Delta U d^{2} z .
$$

One can also define the logarithmic moment $v_{0}$ :

$$
v_{0}=\frac{1}{4 \pi} \iint_{\mathrm{D}} \log |z|^{2} \Delta U(z, \bar{z}) d^{2} z
$$

Again, this integral representation implies that $\Delta U$ is integrable everywhere in $\mathrm{D}$, otherwise a cut-off is required. Similar to $t_{0}$, the moment $v_{0}$ is real. In the important case when $U(z, \bar{z})$ depends only on $z \bar{z}$, i.e., $z \partial_{z} U=\bar{z} \partial_{\bar{z}} U$, the logarithmic moment $v_{0}$ can be equivalently represented as the contour integral

$$
v_{0}=\frac{1}{2 \pi i} \oint_{\gamma}\left(\log |z|^{2} \partial_{z} U-z^{-1} U\right) d z
$$

Let $S(z)$ be the analytic continuation of the function $\partial_{z} U(z, \bar{z})$ away from the contour $\gamma$, then $S(z)=S_{+}(z)+S_{-}(z)$, where $S_{ \pm}(z)$ are analytic functions in D and $\mathrm{D}^{c}$ respectively. They are given by the following integrals of the Cauchy type:

$$
\begin{aligned}
& S_{+}(z)=\frac{1}{2 \pi i} \oint_{\gamma} \frac{\partial_{\zeta} U(\zeta, \bar{\zeta}) d \zeta}{\zeta-z}=\sum_{k \geq 1} k t_{k} z^{k-1}, \quad z \in \mathrm{D} \\
& S_{-}(z)=\frac{1}{2 \pi i} \oint_{\gamma} \frac{\partial_{\zeta} U(\zeta, \bar{\zeta}) d \zeta}{z-\zeta}=\frac{t_{0}}{z}+\sum_{k \geq 1} v_{k} z^{-k-1}, \quad z \in \mathrm{D}^{\mathrm{c}} .
\end{aligned}
$$

From these formulas it follows that $M(z)=z S(z)$. 


\subsection{The dispersionless tau-function}

As is shown in [5]-[7] (see also [10] for the case $\Delta U(z, \bar{z}) \neq$ const), there exists a realvalued function $F_{0}=F_{0}\left(t_{0},\left\{t_{k}\right\},\left\{\bar{t}_{k}\right\}\right)$ such that

$$
d F_{0}=v_{0} d t_{0}+\sum_{k \geq 1}\left(v_{k} d t_{k}+\bar{v}_{k} d \bar{t}_{k}\right)
$$

i.e.,

$$
v_{k}=\frac{\partial F_{0}}{\partial t_{k}}, \quad k \geq 0 .
$$

It is called the dispersionless tau-function and admits the following representation as a double integral over the domain D:

$$
F_{0}=-\frac{1}{16 \pi^{2}} \iint_{\mathrm{D}} \iint_{\mathrm{D}} \Delta U(z, \bar{z}) \log \left|z^{-1}-\zeta^{-1}\right| \Delta U(\zeta, \bar{\zeta}) d^{2} z d^{2} \zeta .
$$

The dispersionless tau-function can be also written as

$$
2 F_{0}=-\frac{1}{4 \pi} \iint_{\mathrm{D}} U \Delta U d^{2} z+t_{0} v_{0}+\sum_{k \geq 1}\left(t_{k} v_{k}+\bar{t}_{k} \bar{v}_{k}\right) .
$$

It satisfies the dispersionless Hirota equations (1.4), (1.5). The conformal map $w(z)$, inverse to the $z(w)$, can be expressed through the dispersionless tau-function as follows:

$$
w(z)=z e^{-\frac{1}{2} \partial_{t_{0}}^{2} F_{0}-\partial_{t_{0}} D(z) F_{0}}, \quad D(z):=\sum_{k \geq 1} \frac{z^{-k}}{k} \partial_{t_{k}} .
$$

Remark. As it was pointed out in [7] (see also [12, 13] and references therein), $F_{0}$ is the free energy of the model of $N \times N$ normal random matrices with the potential $2 \mathcal{R} e \sum_{k} t_{k} z^{k}-U(z, \bar{z})$ in the $N \rightarrow \infty$ limit. In terms of the eigenvalues, the partition function is given by the following $N$-fold integral over the complex plane:

$$
\tau_{N}\left(\left\{t_{j}\right\},\left\{\bar{t}_{j}\right\}\right)=\frac{1}{N !} \int_{\mathbb{C}} \ldots \int_{\mathbb{C}} \prod_{m<n}\left|z_{m}-z_{n}\right|^{2} \prod_{j=1}^{N} e^{-\frac{1}{\hbar} U(z, \bar{z})+\frac{1}{\hbar} \sum_{k \geq 1}\left(t_{k} z_{j}^{k}+\bar{t}_{k} \bar{z}_{j}^{k}\right)} d^{2} z_{j} .
$$

It is known that $\tau_{N}$ is, for any $U(z, \bar{z})$, the tau-function of the 2D Toda lattice hierarchy. Under certain assumptions about the potential $U(z, \bar{z})$, in the limit $\hbar \rightarrow 0, N \rightarrow \infty$ such that $t_{0}=N \hbar$ is fixed, the function

$$
F_{0}=F_{0}\left(t_{0},\left\{t_{j}\right\},\left\{\bar{t}_{j}\right\}\right)=\lim _{\hbar \rightarrow 0}\left(\hbar^{2} \log \tau_{N}\left(\left\{t_{j}\right\},\left\{\bar{t}_{j}\right\}\right)\right)
$$

is the dispersionless tau-function given by (2.26). A matrix model representation of the form (2.29) for the Hurwitz tau-function with the potential $U(z, \bar{z}) \propto(\log (z \bar{z}))^{2}$ (see below) was recently suggested in [25]. 


\subsection{Example: Laplacian growth in radial geometry}

The simplest but important example is $U(z, \bar{z})=z \bar{z}$ which corresponds to the canonical transformation $\bar{z}=z^{-1} M, \bar{M}=M$ (i.e., $M=\bar{M}=z \bar{z}$ ). The string equation (2.14) acquires the form

$$
\left\{z(w), \bar{z}\left(w^{-1}\right)\right\}=1 .
$$

In this case the normal velocity is given by

$$
V_{n}(z)=\frac{1}{2}\left|w^{\prime}(z)\right|, \quad z \in \gamma
$$

Note that $\left|w^{\prime}(z)\right|$ is equal to the normal derivative $\partial_{n} \log |w(z)|$ of the solution to the Laplace equation with a source at infinity and the Dirichlet boundary condition on the contour. Hence (2.32) is identical to the Darcy law for the dynamics of interface between viscous and non-viscous fluids confined in the radial Hele-Shaw cell, assuming that there is no surface tension at the interface. In this way we obtain the exterior LG problem in which the viscous fluid occupies the non-compact exterior domain with a source or sink at infinity. Formulas (2.18) or (2.20) state that

$$
t_{k}=\frac{1}{2 \pi i k} \oint_{\gamma} z^{-k} \bar{z} d z=-\frac{1}{\pi k} \iint_{\mathrm{Dc}^{c}} z^{-k} d^{2} z
$$

are harmonic moments of the exterior of the contour $\gamma$. Their conservation in the Laplacian growth dynamics was established by S.Richardson [30]. Eq. (2.18) states that the time variable $t_{0}=t$ should be identified with area (divided by $\pi$ ) of the compact interior domain encircled by $\gamma$. The function $S(z)$ is the Schwarz function of the contour $\gamma$ [33]. The dispersionless tau-function obeys the quasi-homogeneity condition [7]:

$$
4 F_{0}==-t_{0}^{2}+2 t_{0} v_{0}+\sum_{k \geq 1}(2-k)\left(t_{k} v_{k}+\bar{t}_{k} \bar{v}_{k}\right)
$$

with $v_{k}=\partial_{t_{k}} F_{0}$. Also the relation $\sum_{k \geq 1} k t_{k} v_{k}=\sum_{k \geq 1} k \bar{t}_{k} \bar{v}_{k}$ holds.

A more general example is $U(z, \bar{z})=(z \bar{z})^{1 / N}$ with $N \in \mathbb{Z}$. As it was pointed out

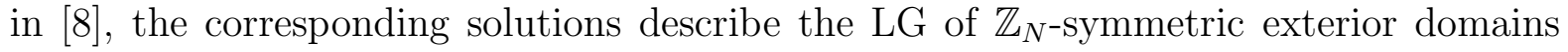
(i.e., symmetric under rotations through the angle $2 \pi / N$ ), or, what is equivalent, the LG in a cone. At negative integer values of $N$ one obtains the interior LG problems. In particular, $N=-1$ corresponds to the Hele-Shaw evolution of a compact domain with a point-like source or sink inside it.

In the next section we consider another important example which corresponds to the Laplacian growth in an infinite channel with periodic boundary conditions in the transverse direction (i.e., in an infinite cylinder). Formally it is as a limiting case $N \rightarrow$ $\infty$ of the $\mathbb{Z}_{N}$-symmetric solutions discussed above but the limit is rather tricky. An independent approach is suggested below. 


\section{Laplacian growth in channel geometry}

\subsection{The moving boundary value problem}

As is described in the introduction, the LG problem in the infinite cylinder (Fig. 2) is translated to the following moving boundary value problem:

$$
\left\{\begin{array}{l}
\Delta \Phi(Z)=0 \quad \text { in } \quad \mathrm{D}_{+} \\
\Phi(Z+2 \pi i R)=\Phi(Z) \\
\Phi(Z)=0, \quad Z \in \Gamma \\
\Phi(Z)=-\frac{1}{2} \mathcal{R} e Z+\ldots \quad \text { as } \mathcal{R} e Z \rightarrow+\infty .
\end{array}\right.
$$

The last condition means that far away to the right from the interface the visocous fluid moves with constant velocity $\vec{V}=\left(\frac{1}{2}, 0\right)$ (in our units time has dimension of length, so the velocity is dimensionless).

Let $W(Z)$ be the conformal map from $\mathrm{D}_{+}$to the right half of the mathematical $W$ plane factorized over shifts by $2 \pi i$ such that $W(Z+2 \pi i R)=W(Z)+2 \pi i$ with the expansion as $\mathcal{R} e Z \rightarrow+\infty$ of the form

$$
W(Z)=Z / R+\sum_{k \geq 0} c_{k} e^{-k Z / R}
$$

Then the solution to the boundary value problem (3.1) is given by $\Phi(Z)=-\frac{R}{2} \mathcal{R} e W(Z)$. Since $\partial_{n} \mathcal{R} e W(Z)=\left|W^{\prime}(Z)\right|$ for all $z \in \Gamma$, the normal velocity is

$$
V_{n}(Z)=\frac{R}{2}\left|W^{\prime}(Z)\right|
$$

The inverse conformal map, $Z(W)$, is given by the series of the form

$$
Z(W)=R W+\sum_{k \geq 0} u_{k} e^{-k W} .
$$

The Laplacian growth equation (1.2) for $Z(W)$ can be derived with the help of the same kinematic identity (2.15) applied to the contour in the $Z$-plane with $\sigma=-i W$, then $\partial \sigma / \partial l=\left|W^{\prime}(Z)\right|$, and the identity combined with the Darcy law yields

$$
\frac{\partial Z(W)}{\partial W} \frac{\partial \bar{Z}(-W)}{\partial t}-\frac{\partial Z(W)}{\partial t} \frac{\partial \bar{Z}(-W)}{\partial W}=R
$$

or, in terms of the Poisson bracket, $\{Z(W), \bar{Z}(-W)\}_{W, t}=R$.

A remark on the LG problem in an infinite channel with rigid walls is in order. In this case instead of periodicity condition $\Phi(Z+2 \pi i R)=\Phi(Z)$ one should impose the no-flux conditions $\partial_{Y} \Phi(Z)=\partial_{Y} \Phi(Z+2 \pi i R)=0$ on the walls $Z=X$ (the real line) and $Z=X+2 \pi i R$ (the line $Y=2 \pi R$ ). In particular, this implies that the tangent lines to the interface at the endpoints are orthogonal to the walls. This problem can be formally reduced to the problem in a cylinder of radius $2 R$ with the additional $\mathbb{Z}_{2}$ reflection symmetry $Y \rightarrow-Y$ which in the complex coordinates is the complex conjugation $Z \rightarrow \bar{Z}$. This symmetry implies that the coefficients $u_{k}$ in (3.4) should be real. 

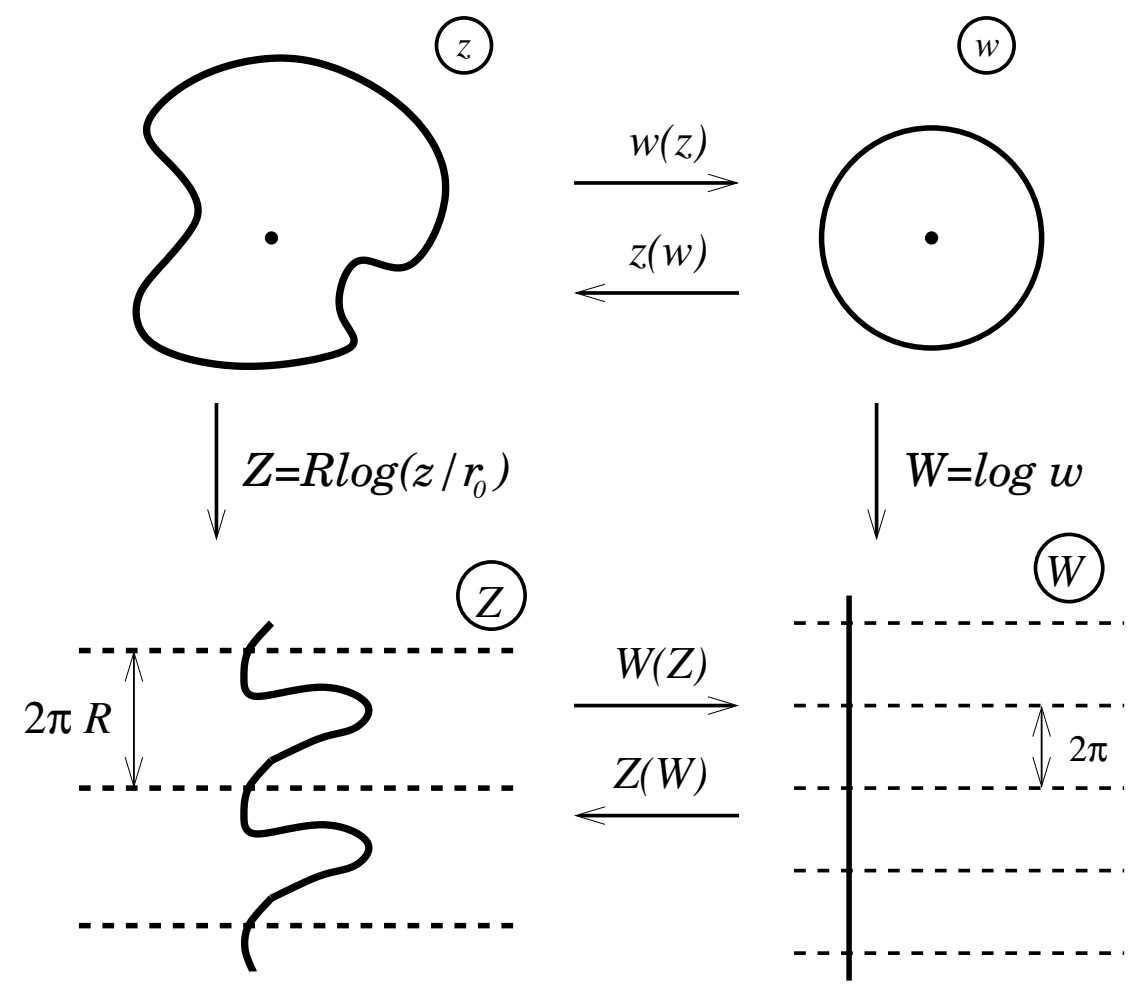

Figure 4: The auxiliary and real "physical" and "mathematical" planes.

\subsection{A growth problem in the auxiliary physical plane}

Our strategy will be to map this problem to the auxiliary physical plane (the $z$-plane), where it becomes a radial growth problem of the type discussed above (Fig. (4). This is achieved by the conformal transformation

$$
z=r_{0} e^{Z / R} \quad \text { or } \quad Z=R \log \left(z / r_{0}\right),
$$

where $r_{0}$ is some (time-independent) scale in the $z$-plane. The conformal maps $Z(W)$ and $z(w)$ are thus related by the formulas

$$
\begin{gathered}
z(w)=r_{0} e^{Z(\log w) / R}, \quad w(z)=e^{W\left(R \log \left(z / r_{0}\right)\right)}, \\
Z(W)=R \log \left(\frac{z\left(e^{W}\right)}{r_{0}}\right), \quad W(Z)=\log w\left(r_{0} e^{Z / R}\right) .
\end{gathered}
$$

The contour $\Gamma$ is mapped to the Lax contour $\gamma$, and their normal velocities, $V_{n}=V_{n}^{(Z)}$ and $V_{n}^{(z)}$, are connected by the formula

$$
V_{n}^{(Z)}=\left|\frac{d Z}{d z}\right| V_{n}^{(z)}=\frac{R}{|z|} V_{n}^{(z)} .
$$

The domains $D_{+}$and $D_{+}$in the physical plane are mapped to $D^{c}$ and $D$ respectively in the auxiliary physical plane. The $X=0$ section of the cylinder is mapped to the circle $|z|=r_{0}$, so our assumption means that the domain $\mathrm{D}$ contains the disk $|z| \leq r_{0}$. 
According to the general method outlined in Section 2.2, let us take the following generating function of the canonical transformation:

$$
U(z, \bar{z})=\frac{R}{2}\left[\log \frac{z \bar{z}}{r_{0}^{2}}\right]^{2} .
$$

We consider it as a function on the auxiliary physical plane. Then

$$
M=\bar{M}=z \partial_{z} U=\bar{z} \partial_{\bar{z}} U=R \log \frac{z \bar{z}}{r_{0}^{2}}
$$

or, equivalently,

$$
z \bar{z}=r_{0}^{2} e^{M / R}
$$

and

$$
\partial_{z} \partial_{\bar{z}} U=\frac{R}{z \bar{z}} .
$$

The normal velocity of the Lax contour in the $z$-plane is

$$
V_{n}^{(z)}(z)=\frac{|z|^{2}}{2 R}\left|w^{\prime}(z)\right|, \quad z \in \gamma
$$

Using (3.6) and $\left|w^{\prime}(z)\right|=\frac{R}{|z|}\left|W^{\prime}(Z)\right|$, we can find the normal velocity $V_{n}^{(Z)}$ in the corresponding point of the contour $\Gamma$ :

$$
V_{n}^{(Z)}(Z)=\frac{R}{|z|} V_{n}^{(z)}(z)=\frac{R}{2}\left|W^{\prime}(Z)\right|
$$

which coincides with (3.3) . The string equation (2.14) in the auxiliary physical plane reads

$$
\left\{z(w), \bar{z}\left(w^{-1}\right)\right\}=R^{-1} z(w) \bar{z}\left(w^{-1}\right) .
$$

After the change of variables $z(w)=r_{0} e^{Z(W) / R}, \bar{z}\left(w^{-1}\right)=r_{0} e^{\bar{Z}(-W) / R}$ it becomes the Laplacian growth equation (3.5) in the physical plane. This proves the isomorphism between the radial growth problem in the $z$-plane and the physical problem on the cylinder.

It remains to identify the time variables (deformation parameters) with moments of $\mathrm{D}_{+}$. The time $t$ is identified with the $t_{0}$-variable. In the case at hand the density $\Delta U(z, \bar{z})=R /|z|^{2}$ is non-integrable at $z=0$, so some modifications in formulas from the previous section are necessary. Let $\mathrm{B}\left(r_{0}\right)$ be the disk of radius $r_{0}$ centered at the origin, then in the $z$-plane we have

$$
t_{0}=\frac{R}{\pi i} \oint_{\gamma} \log \left(\frac{|z|}{r_{0}}\right) \frac{d z}{z}=\frac{R}{\pi} \iint_{\mathrm{D} \backslash \mathrm{B}\left(r_{0}\right)} \frac{d^{2} z}{z \bar{z}} .
$$

Equivalently, in the $Z$-plane these integrals are represented as

$$
t_{0}=\frac{1}{2 \pi i R} \int_{\Gamma}(Z+\bar{Z}) d Z=\frac{1}{\pi R} \int_{\Gamma} X d Y=\frac{\operatorname{Area}\left(\mathrm{D}_{-}^{(0)}\right)}{\pi R},
$$

where Area $\left(D_{-}^{(0)}\right)$ is the area of the domain $D_{-}^{(0)}$ bounded by the curve $\Gamma$ and the section $X=0$. Note that $\mathrm{D}_{-}^{(0)}$ is the image of $\mathrm{B}\left(r_{0}\right)$ under the map from the auxiliary physical plane. The higher times $t_{k}$ (integrals of motion for the Laplacian growth) are

$$
t_{k}=\frac{R}{\pi i k} \oint_{\gamma} z^{-k} \log \left(\frac{|z|}{r_{0}}\right) \frac{d z}{z}=\frac{r_{0}^{-k}}{\pi i k R} \int_{\Gamma} e^{-k Z / R} X d Z=-\frac{r_{0}^{-k}}{\pi k R} \iint_{\mathrm{D}_{+}} e^{-k Z / R} d^{2} Z .
$$


The complimentary moments (dynamical variables for the Laplacian growth) are

$$
v_{k}=\frac{R}{\pi i} \oint_{\gamma} z^{k} \log \left(\frac{|z|}{r_{0}}\right) \frac{d z}{z}=\frac{r_{0}^{k}}{\pi i R} \int_{\Gamma} e^{k Z / R} X d Z=\frac{r_{0}^{k}}{\pi R} \iint_{\mathrm{D}_{-}} e^{k Z / R} d^{2} Z
$$

For $k \geq 1$ no regularization is required and, as it is clearly seen from the contour integral formulas in the $z$-plane, the $t_{k}$ 's and $v_{k}$ 's do not depend on $r_{0}$. However, the integral (2.22) for the logarithmic moment $v_{0}$ diverges. One should cut off the integral at $|z|=r_{0}$ :

$$
v_{0}=\frac{R}{\pi} \iint_{\mathrm{D} \backslash \mathrm{B}\left(r_{0}\right)} \frac{\log (z \bar{z})}{z \bar{z}} d^{2} z=2 t_{0} \log r_{0}+\frac{2}{\pi R^{2}} \int_{\mathrm{D}_{-}^{(0)}} X d^{2} Z .
$$

An important relation between the moments can be derived by calculation of the integral $I=\frac{1}{2 \pi i} \oint_{\gamma} U(z, \bar{z}) z^{-1} d z$ with $U$ given by (3.7) in two different ways. First, by the Stokes formula,

$$
I=\frac{1}{\pi} \iint_{\mathrm{D} \backslash \mathrm{B}\left(r_{0}\right)} \bar{z} \partial_{\bar{z}} U \frac{d^{2} z}{z \bar{z}}=\frac{R}{\pi} \iint_{\mathrm{D} \backslash \mathrm{B}\left(r_{0}\right)} \log \left|z / r_{0}\right|^{2} \frac{d^{2} z}{|z|^{2}}=v_{0}-2 t_{0} \log r_{0} .
$$

On the other hand, comparing (3.7) and (3.8), we see that for $z \in \gamma$ it holds

$$
U(z, \bar{z})=\frac{M^{2}(z)}{2 R}
$$

SO

$$
I=\frac{1}{4 \pi i R} \oint_{\gamma} M^{2}(z) \frac{d z}{z}=\frac{1}{4 \pi i R} \oint_{\gamma}\left(\sum_{k \geq 1} k t_{k} z^{k}+t_{0}+\sum_{k \geq 1} v_{k} z^{-k}\right)^{2} \frac{d z}{z}=\frac{t_{0}^{2}}{2 R}+\frac{1}{R} \sum_{k \geq 1} k t_{k} v_{k} .
$$

Equating the results, we obtain the identity

$$
R v_{0}=\frac{t_{0}^{2}}{2}+2 R t_{0} \log r_{0}+\sum_{k \geq 1} k t_{k} v_{k}
$$

\subsection{The tau-function}

The dispersionless tau-function is given by the double integral in the $z$-plane over $\mathrm{D}(2.26)$. However, in our case the integral diverges at small $|z|,|\zeta|$ and one should introduce a cut-off:

$$
F_{0}=-\frac{R^{2}}{\pi^{2}} \iint_{\mathrm{D} \backslash \mathrm{B}\left(r_{0}\right)} \iint_{\mathrm{D} \backslash \mathrm{B}\left(r_{0}\right)} \log \left|z^{-1}-\zeta^{-1}\right| \frac{d^{2} z d^{2} \zeta}{|z \zeta|^{2}} .
$$

The same cut-off should be introduced in (2.27):

$$
2 F_{0}=-\frac{2 R^{2}}{\pi} \iint_{\mathrm{D} \backslash \mathrm{B}\left(r_{0}\right)}\left[\log \left(|z| / r_{0}\right)\right]^{2} \frac{d^{2} z}{|z|^{2}}+t_{0} v_{0}+\sum_{k \geq 1}\left(t_{k} v_{k}+\bar{t}_{k} \bar{v}_{k}\right) .
$$

In the physical plane these integrals are written as

$$
F_{0}=-\frac{1}{\pi^{2} R^{2}} \iint_{\mathrm{D}_{-}^{(0)}} \iint_{\mathrm{D}_{-}^{(0)}} \log \left|e^{-Z / R}-e^{-Z^{\prime} / R}\right| d^{2} Z d^{2} Z^{\prime}-t_{0}^{2} \log r_{0},
$$




$$
2 F_{0}=-\frac{2}{\pi R^{2}} \iint_{\mathrm{D}_{-}^{(0)}} X^{2} d^{2} Z+t_{0} v_{0}+\sum_{k \geq 1}\left(t_{k} v_{k}+\bar{t}_{k} \bar{v}_{k}\right) .
$$

The general formulas $v_{k}=\partial F_{0} / \partial t_{k}(2.25)$ hold in this case as well, so equation (3.20) is equivalent to the following relation for the first order derivatives of the function $F_{0}$ :

$$
\frac{\partial F_{0}}{\partial t_{0}}=\frac{t_{0}^{2}}{2 R}+2 t_{0} \log r_{0}+\frac{1}{R} \sum_{k \geq 1} k t_{k} \frac{\partial F_{0}}{\partial t_{k}} .
$$

The integral in (3.22) can be simplified using the identity

$$
\frac{1}{4 \pi} \iint_{\mathrm{D} \backslash \mathrm{B}\left(r_{0}\right)} U \Delta U d^{2} z=\frac{1}{2 \pi i} \oint_{\partial \mathrm{D}} U \partial_{z} U d z-\frac{1}{2 \pi i} \oint_{\partial \mathrm{B}\left(r_{0}\right)} U \partial_{z} U d z-\frac{1}{\pi} \iint_{\mathrm{D} \backslash \mathrm{B}\left(r_{0}\right)}\left|\partial_{z} U\right|^{2} d^{2} z
$$

valid for any domain $\mathrm{D}$ in the $z$-plane and any $U$ regular in $\mathrm{D} \backslash \mathrm{B}\left(r_{0}\right)$. We notice that in our case $\left|\partial_{z} U\right|^{2}=\frac{1}{2} U \Delta U$ and $U=\partial_{z} U=0$ on $\partial \mathrm{B}\left(r_{0}\right)$ (i.e., at $|z|=r_{0}$ ), hence

$$
\frac{1}{4 \pi} \iint_{\mathrm{D}} U \Delta U d^{2} z=\frac{1}{6 \pi i} \oint_{\gamma} U \partial_{z} U d z=\frac{1}{6 \pi i} \oint_{\gamma} U(z, \bar{z}) S(z) d z .
$$

Using (3.19), we can rewrite the r.h.s. entirely in terms of $M(z)$, so

$$
\frac{1}{4 \pi} \iint_{\mathrm{D}} U \Delta U d^{2} z=\frac{1}{12 \pi i R} \oint_{\gamma} M^{3}(z) \frac{d z}{z}
$$

Plugging here the series (2.11) for $M(z)$ and extracting the residues, we find:

$$
\frac{1}{4 \pi} \iint_{\mathrm{D}} U \Delta U d^{2} z=\frac{t_{0}^{3}}{6 R}+\frac{t_{0}}{R} \sum_{k \geq 1} k t_{k} v_{k}+\frac{1}{2 R} \sum_{k, l \geq 1}\left(k l t_{k} t_{l} v_{k+l}+(k+l) t_{k+l} v_{k} v_{l}\right) .
$$

(Note that the expression in the right hand side must be real although it can not be directly seen from its form.) Therefore, equation (3.22) takes the form

$$
\begin{aligned}
2 F_{0} & =t_{0} v_{0}+\sum_{k \geq 1}\left(t_{k} v_{k}+\bar{t}_{k} \bar{v}_{k}\right)-\frac{t_{0}^{3}}{6 R}-\frac{t_{0}}{R} \sum_{k \geq 1} k t_{k} v_{k} \\
& -\frac{1}{2 R} \sum_{k, l \geq 1}\left(k l t_{k} t_{l} v_{k+l}+(k+l) t_{k+l} v_{k} v_{l}\right) .
\end{aligned}
$$

It can be further simplified using relation (3.20):

$$
F_{0}=\frac{t_{0}^{3}}{6 R}+t_{0}^{2} \log r_{0}+\frac{1}{2} \sum_{k \geq 1}\left(t_{k} v_{k}+\bar{t}_{k} \bar{v}_{k}\right)-\frac{1}{4 R} \sum_{k, l \geq 1}\left(k l t_{k} t_{l} v_{k+l}+(k+l) t_{k+l} v_{k} v_{l}\right) .
$$

Let us examine how this function depends on $r_{0}$ and $R$. As it follows from (3.16), (3.17), the moments $t_{k}, v_{k}$ with $k \geq 1$ do not depend on $r_{0}$. The dependence on $r_{0}$ comes from the cut-off at small distances of the formally divergent integrals for $t_{0}, v_{0}$ and the integral in (3.22). It is not difficult to see that

$$
\frac{d}{d \log r_{0}}\left(\frac{1}{\pi} \iint_{\mathrm{D} \backslash \mathrm{B}\left(r_{0}\right)} \frac{\left(\log |z|^{2}\right)^{k}}{|z|^{2}} d^{2} z\right)=-2^{k+1}\left(\log r_{0}\right)^{k} .
$$


In particular, $d t_{0} / d \log r_{0}=-2 R, d v_{0} / d \log r_{0}=-4 R \log r_{0}$. The full derivative of (3.22) is then easily calculated to be

$$
\frac{d F_{0}}{d \log r_{0}}=-4 R t_{0} \log r_{0}
$$

Since $\frac{d}{d \log r_{0}}\left(\frac{t_{0}^{3}}{6 R}+t_{0}^{2} \log r_{0}\right)=-4 R t_{0} \log r_{0}$, we see that the function

$$
\tilde{F}_{0}=F_{0}-\frac{t_{0}^{3}}{6 R}-t_{0}^{2} \log r_{0}
$$

does not depend on $r_{0}$ :

$$
\frac{d \tilde{F}_{0}}{d \log r_{0}}=\frac{\partial \tilde{F}_{0}}{\partial \log r_{0}}+\frac{\partial \tilde{F}_{0}}{\partial t_{0}} \frac{d t_{0}}{\partial \log r_{0}}=\frac{\partial \tilde{F}_{0}}{\partial \log r_{0}}-2 R \frac{\partial \tilde{F}_{0}}{\partial t_{0}}=0 .
$$

The last equality means that the function $\tilde{F}_{0}$ depends on $r_{0}$ and $t_{0}$ only in the combination $t_{0}+2 R \log r_{0}\left(\right.$ or $\left.r_{0}^{2} e^{t_{0} / R}\right)$.

The derivative $\partial F_{0} / \partial R$ at fixed $t_{k}$ can be found by the general variational method [7, 10] but in our case a simpler argument works. First we pass to the dimensionless times $\hat{t}_{k}=t_{k} / R$, then the new times $\hat{t}_{k}$ are $R$-independent. As is seen from equation (3.21), $F_{0}$ is of the form $F_{0}=R^{2} \hat{F}_{0}$, where $\hat{F}_{0}$ is $R$-independent. Therefore, we can write

$$
F_{0}\left(R,\left\{t_{k}\right\}\right)=F_{0}\left(R,\left\{R \hat{t}_{k}\right\}\right)=R^{2} \hat{F}=R^{2} F_{0}\left(1,\left\{\hat{t}_{k}\right\}\right)
$$

Next, taking the total $R$-derivative of the identity $R^{-2} F_{0}\left(R,\left\{R \hat{t}_{k}\right\}\right)=F_{0}\left(1,\left\{\hat{t}_{k}\right\}\right)$, we find:

$$
-2 R^{-3} F_{0}+R^{-2} \partial_{R} F_{0}+R^{-2}\left(\hat{t}_{0} \partial_{t_{0}} F_{0}+2 \mathcal{R} e \sum_{k \geq 1} \hat{t}_{k} \partial_{t_{k}} F_{0}\right)=0
$$

or

$$
2 F_{0}=R \partial_{R} F_{0}+t_{0} \partial_{t_{0}} F_{0}+\sum_{k \geq 1}\left(t_{k} \partial_{t_{k}} F_{0}+\bar{t}_{k} \partial_{\bar{t}_{k}} F_{0}\right),
$$

where the partial derivative $\partial_{R}$ is taken at fixed $t_{k}$ and the derivatives $\partial_{t_{k}}$ are taken at fixed $R$. Comparing with (3.27) and taking into account that $v_{k}=\partial_{t_{k}} F_{0}$, we conclude that

$$
-R^{2} \partial_{R} F_{0}=\frac{\partial F_{0}}{\partial R^{-1}}=\frac{t_{0}^{3}}{6}+t_{0} \sum_{k \geq 1} k t_{k} v_{k}+\frac{1}{2} \sum_{k, l \geq 1}\left(k l t_{k} t_{l} v_{k+l}+(k+l) t_{k+l} v_{k} v_{l}\right) .
$$

Using the notation $\beta=1 / R$, as in [27, we rewrite this equality in the form

$$
\partial_{\beta} F_{0}=\frac{t_{0}^{3}}{6}+t_{0} \sum_{k \geq 1} k t_{k} \partial_{t_{k}} F_{0}+\frac{1}{2} \sum_{k, l \geq 1}\left(k l t_{k} t_{l} \partial_{t_{k+l}} F_{0}+(k+l) t_{k+l} \partial_{t_{k}} F_{0} \partial_{t_{l}} F_{0}\right)
$$

which agrees with the formula given in [27]. The term with the double sum comes from the action of the "cut-and-join operator" in the limit of zero dispersion (more details are in the next section). 
Passing in general formula (2.28) for the conformal map $w(z)$ to the variables $W, Z$, we obtain

$$
W(Z)=Z / R+\log r_{0}-\frac{1}{2} \partial_{t_{0}}^{2} F_{0}-\sum_{k \geq 1} \frac{r_{0}^{-k}}{k} e^{-k Z / R} \partial_{t_{k}} \partial_{t_{0}} F_{0} .
$$

Here we recoginize equation (1.6) from the introduction (written there at $r_{0}=1$ ).

Finally, we note that in the limit $R \rightarrow \infty(\beta=0)$ the function $F_{0}$ can be found explicitly:

$$
\left.F_{0}\right|_{\beta=0}=t_{0}^{2} \log r_{0}+\sum_{k \geq 1} k r_{0}^{2 k} t_{k} \bar{t}_{k}
$$

To see this, we take into account that our independent variables $t_{k}$ must be kept finite in this limit. The first integral formula in (3.16) implies that the contour $\gamma$ has to be close to the circle $|z|=r_{0}$. More precisely, the contour has to be of the form

$$
z(\theta)=r_{0}\left(1+\frac{f(\theta)}{R}\right) e^{i \theta}+O\left(R^{-2}\right), \quad 0 \leq \theta<2 \pi
$$

with some real-valued $2 \pi$-periodic function $f$. Then in the limit $R \rightarrow \infty$ we get

$$
t_{k}=\frac{r_{0}^{-k}}{\pi k} \int_{0}^{2 \pi} f(\theta) e^{-i k \theta} d \theta
$$

while the same limit in the integral representation of $v_{k}(3.17)$ yields

$$
v_{k}=\frac{r_{0}^{k}}{\pi k} \int_{0}^{2 \pi} f(\theta) e^{i k \theta} d \theta
$$

so $v_{k}=k r_{0}^{2 k} \bar{t}_{k}$. Plugging this into (3.28) with $R \rightarrow \infty$, we obtain (3.34).

Remark. For solutions with the reflection $\mathbb{Z}_{2}$-symmetry $Z \rightarrow \bar{Z}$ (which describe Hele-Shaw flows in a channel with rigid walls) all the moments $t_{k}$ are constrained to be real numbers, i.e., $t_{k}=\bar{t}_{k}$. However, the vector fields $\partial_{t_{k}}$ and $\partial_{\bar{t}_{k}}$ of the dToda flows are transversal to the real submanifold defined by the conditions $t_{k}=\bar{t}_{k}$. This means that in general the dToda hierarchy can not be restricted to the class of solutions with reflection symmetry.

\subsection{Example: the case of non-zero $t_{0}, t_{1}$ (trochoid)}

The simplest possible case is when all moments except $t_{0}$ are zero: $t_{0}=t, t_{k}=0$ at $k \geq 1$. It corresponds to the uniform motion of the circular section $X=$ const of the cylinder with velocity $\frac{1}{2}: X(t, \sigma)=t / 2, Y(t, \sigma)=R \sigma$. In this case $v_{0}=\frac{t_{0}^{2}}{2 R}+2 t_{0} \log r_{0}$ and the tau-function is

$$
F_{0}=\frac{t_{0}^{3}}{6 R}+t_{0}^{2} \log r_{0}
$$

Next in order of complexity is the case $t_{0}=t, t_{1} \neq 0, t_{k}=0$ at $k \geq 2$. As is readily seen from (3.16), if only the first $N$ moments are non-zero, then the series (3.4) truncates at the $N$-th term. In particular, in our case we have:

$$
Z(W)=R W+u_{0}+u_{1} e^{-W}, \quad \bar{Z}(-W)=-R W+\bar{u}_{0}+\bar{u}_{1} e^{W}
$$


in the physical plane or

$$
z(w)=r w e^{\tilde{u}_{1} w^{-1}}, \quad r=r_{0} e^{u_{0} / R}, \quad \tilde{u}_{1}=u_{1} / R
$$

in the auxiliary physical plane (note that the latter equation has the form of the Lambert curve $x=y e^{y}$ for $z^{-1}$ and $w^{-1}$ ). Plugging this into the LG equation (3.5), we get two conditions, one real and one complex:

$$
\left\{\begin{array}{l}
2 R \mathcal{R} \text { e } \dot{u}_{0}-\partial_{t}\left|u_{1}\right|^{2}=R \\
R \dot{u}_{1}=u_{1} \dot{\bar{u}}_{0} .
\end{array}\right.
$$

They can be easily integrated:

$$
u_{1}=R \kappa e^{\left(\bar{u}_{0}+i Y_{0}\right) / R}, \quad \frac{u_{0}+\bar{u}_{0}}{R}-\kappa^{2} e^{\left(u_{0}+\bar{u}_{0}\right) / R}=\frac{t}{R} .
$$

Here the real parameters $0<\kappa<1, Y_{0}$ come from a complex integration constant. Set $R \lambda^{2}=u_{0}+\bar{u}_{0}-t$, and let $\lambda$ be the positive root, then the time-dependent contour is given by

$$
Z(\sigma, t)=i R \sigma+i Y_{0}+\frac{t+R \lambda^{2}(t)}{2}+R \lambda(t) e^{-i \sigma},
$$

where the real $\lambda=\lambda(t)>0$ obeys the equation

$$
\lambda^{2}=\kappa^{2} e^{\lambda^{2}+\frac{t}{R}}
$$

In terms of the Lambert function $\mathbf{W}(x)$ defined by the equation $x=\mathbf{W}(x) e^{\mathbf{W}(x)}$ we can write

$$
\lambda^{2}=-\mathbf{W}\left(-\kappa^{2} e^{t / R}\right)
$$

In the coordinates $X, Y$ the contour is

$$
\begin{aligned}
\frac{X}{R} & =\frac{t}{2 R}+\frac{\lambda^{2}}{2}+\lambda \cos \sigma, \\
\frac{Y-Y_{0}}{R} & =\sigma-\lambda \sin \sigma .
\end{aligned}
$$

At $0<\lambda<1$ it is a trochoid (curtate cycloid, Fig. 5). The initial value $\lambda_{0}=\lambda(0)$ is found as a root of the equation $\lambda_{0}^{2}=\kappa^{2} e^{\lambda_{0}^{2}}$ such that $\lambda_{0} \rightarrow 0$ as $\kappa \rightarrow 0$. From equation (3.35) it follows that $\dot{\lambda}>0$. At $\lambda=1$ which corresponds to the critical value of time $t_{c}=2 R \log \kappa^{-1}-R$, the curtate cycloid becomes the ordinary cycloid (Fig. 6) with a singularity (a cusp) at $\sigma=0$. This is an example of the well known finite time singularities typical for the LG with zero surface tension.

The calculation of the integral (3.16) yields

$$
t_{1}=\frac{R \kappa}{r_{0}} e^{-i Y_{0} / R},
$$

hence $\kappa=r_{0}\left|t_{1}\right| / R$. The higher moments $t_{k}$ vanish. The complimentary moments $v_{k}$ are obtained from (3.20) (or (3.18) ) and (3.17). All of them are in general non-zero. We 


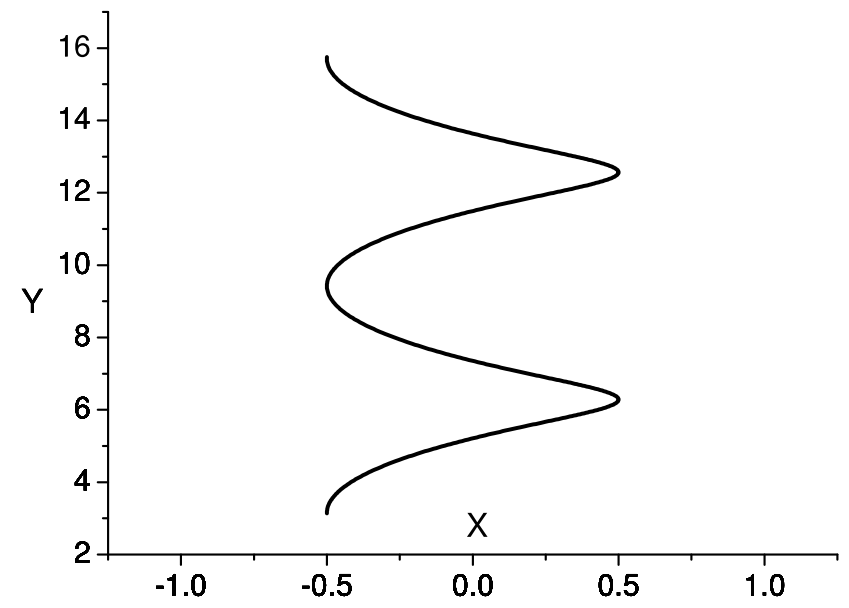

Figure 5: The curtate cycloid $X=\frac{1}{2} \cos \sigma, Y=\sigma-\frac{1}{2} \sin \sigma$.

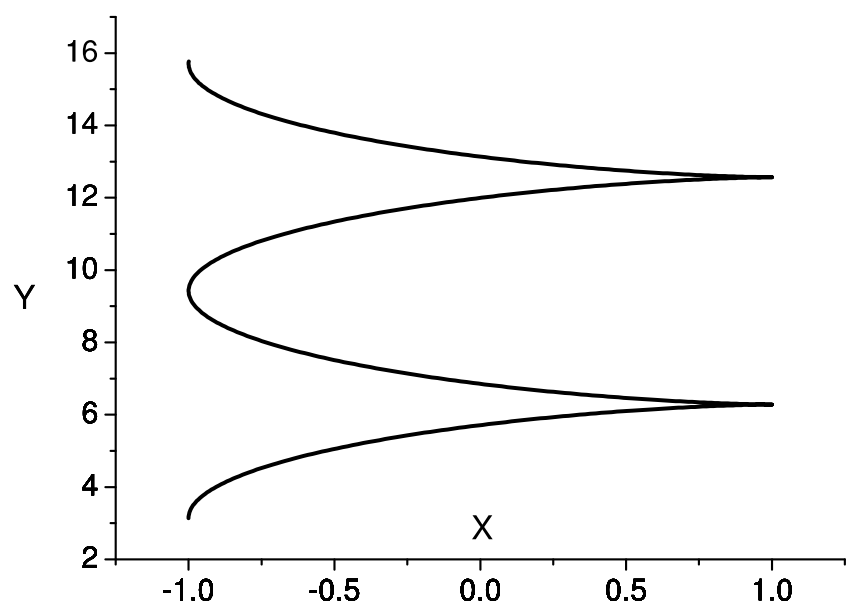

Figure 6: The cycloid $X=\cos \sigma, Y=\sigma-\sin \sigma$.

need them at $k=0,1,2$ :

$$
\begin{aligned}
& v_{0}=2 t_{0} \log r_{0}+\frac{t_{0}^{2}}{2 R}+\frac{R}{2} \lambda^{2}\left(2-\lambda^{2}\right) \\
& v_{1}=\frac{R^{2}}{2 t_{1}} \lambda^{2}\left(2-\lambda^{2}\right) \\
& v_{2}=\frac{R^{3}}{3 t_{1}^{2}} \lambda^{4}\left(3-2 \lambda^{2}\right)
\end{aligned}
$$

The dispersionless tau-function can be found with the help of (3.27) or (3.28). In our case all sums there are finite:

$$
2 F_{0}=t_{0} v_{0}+t_{1} v_{1}+\bar{t}_{1} \bar{v}_{1}-\frac{t_{0}^{3}}{6 R}-\frac{t_{0}}{R} t_{1} v_{1}-\frac{1}{2 R} t_{1}^{2} v_{2}
$$

The last term is the contribution of the cut-and-join part of (3.27). Plugging the values of the moments $v_{k}$ calculated above, we get:

$$
F_{0}=\frac{t_{0}^{3}}{6 R}+t_{0}^{2} \log r_{0}+\frac{R^{2}}{12} \lambda^{2}\left(2 \lambda^{4}-9 \lambda^{2}+12\right),
$$


where $\lambda$ is defined as an implicit function of $t_{0}, t_{1}, \bar{t}_{1}$ by equation (3.35) which in terms of the moments takes the form

$$
R^{2} \lambda^{2}=r_{0}^{2} t_{1} \bar{t}_{1} e^{\lambda^{2}+\frac{t_{0}}{R}}
$$

or $\lambda^{2}=-\mathrm{W}\left(-\left|t_{1}\right|^{2} r_{0}^{2} e^{\frac{t_{0}}{R}} / R^{2}\right)$. This equation allows one to find partial derivatives of the function $\lambda$ :

$$
\partial_{t_{0}} \lambda=\frac{\lambda}{2 R\left(1-\lambda^{2}\right)}, \quad \partial_{t_{1}} \lambda=\frac{\lambda}{2 t_{1}\left(1-\lambda^{2}\right)}, \quad \partial_{R} \lambda=-\frac{\lambda\left(t_{0}+2 R\right)}{2 R^{2}\left(1-\lambda^{2}\right)}
$$

and directly check the formulas $v_{0}=\partial F_{0} / \partial t_{0}, v_{1}=\partial F_{0} / \partial t_{1}$, as well as the first equation of the dToda hierarchy

$$
\partial_{t_{1} \bar{t}_{1}}^{2} F_{0}=e^{\partial_{t_{0}}^{2} F_{0}}
$$

In the limit $R \rightarrow \infty(\beta=0)$ equation (3.38) yields $\lambda \rightarrow 0$ with $R \lambda \rightarrow r_{0}\left|t_{1}\right|$, so from (3.37) we have

$$
\left.F_{0}\right|_{\beta=0}=t_{0}^{2} \log r_{0}+r_{0}^{2} t_{1} \bar{t}_{1} \quad \text { at } \quad t_{k}=0, k \geq 2
$$

which agrees with (3.34).

Note that the $v_{k}$ 's, as well as their derivatives with respect to $t_{0}, t_{1}$, are non-singular at the critical point $\lambda=\lambda_{c}=1$ which corresponds to the critical value of $t_{0}$

$$
t_{0}^{(c)}=2 R \log \left(\frac{R}{r_{0}\left|t_{1}\right|}\right)-R
$$

However, their second derivatives, for example

$$
\frac{\partial^{2} v_{0}}{\partial t_{0}^{2}}=\frac{1}{R\left(1-\lambda^{2}\right)}
$$

are singular at the critical point.

Set $x^{2}=\beta^{2} r_{0}^{2} t_{1} \bar{t}_{1} e^{\beta t_{0}}$, then the Taylor expansion of the function $\lambda^{2}$ as $x \rightarrow 0$ reads

$$
\lambda^{2}=-\mathrm{W}\left(-x^{2}\right)=\sum_{k=1}^{\infty} \frac{k^{k-1}}{k !} x^{2 k}=x^{2}+x^{4}+\frac{3}{2} x^{6}+\frac{8}{3} x^{8}+\ldots
$$

Plugging this into (3.37), we get for the restriction of the function $F_{0}\left(\beta, r_{0}^{2}, t_{0},\left\{t_{k}\right\},\left\{\bar{t}_{k}\right\}\right)$ to the submanifold $t_{j}=0, j \geq 2$,

$$
\begin{aligned}
F_{0}\left(\beta, r_{0}^{2}, t_{0}, t_{1}, \bar{t}_{1}\right)= & \frac{t_{0}^{3}}{6 R}+t_{0}^{2} \log r_{0}+r_{0}^{2} e^{\beta t_{0}} t_{1} \bar{t}_{1}+\frac{1}{4} \beta^{2} r_{0}^{4} e^{2 \beta t_{0}} t_{1}^{2} \bar{t}_{1}^{2} \\
& +\frac{1}{6} \beta^{4} r_{0}^{6} e^{3 \beta t_{0}} t_{1}^{3} \bar{t}_{1}^{3}+\frac{1}{6} \beta^{6} r_{0}^{8} e^{4 \beta t_{0}} t_{1}^{4} \bar{t}_{1}^{4}+\ldots
\end{aligned}
$$

At $t_{0}=0$ we get the series

$$
F_{0}=r_{0}^{2} t_{1} \bar{t}_{1}+\frac{1}{4} \beta^{2} r_{0}^{4} t_{1}^{2} \bar{t}_{1}^{2}+\frac{1}{6} \beta^{4} r_{0}^{6} t_{1}^{3} \bar{t}_{1}^{3}+\frac{1}{6} \beta^{6} r_{0}^{8} t_{1}^{4} \bar{t}_{1}^{4}+\ldots=r_{0}^{2} \sum_{n \geq 1} \frac{n^{n-3}}{n !}\left(\beta r_{0}\right)^{2 n-2} t_{1}^{n} \bar{t}_{1}^{n}
$$

As is explained in the next section, it has the form

$$
\left.F_{0}\right|_{\substack{t_{0}=0 \\ t_{1} \neq 0}}=\sum_{d \geq 1} \frac{r_{0}^{2 d} \beta^{2 d-2}}{(2 d-2) !} H_{d, 2 d-2}\left(1^{d}, 1^{d}\right) t_{1}^{d} \bar{t}_{1}^{d}
$$


where

$$
H_{d, 2 d-2}\left(1^{d}, 1^{d}\right)=\frac{(2 d-2) !}{d !} d^{d-3}
$$

is the number of degree $d$ coverings $\mathbb{C P}^{1} \longrightarrow \mathbb{C P}^{1}$ with exactly $2 d-2$ simple ramification points.

\section{The LG tau-function as the generating function for genus zero double Hurwitz numbers}

As it was already pointed out in the introduction, the dispersionless tau-function $F_{0}$ for the LG in a channel is closely related to the genus-zero part of the generating function for the double Hurwitz numbers which count ramified coverings of $\mathbb{C P}^{1}$ of genus 0 with arbitrary ramification type at two marked points. In this section we outline the precise connection between the LG tau-function (3.21) and the generating function for the double Hurwitz numbers.

The Hurwitz numbers count ramified coverings of $\mathbb{C P}^{1}$. Let $f: \Sigma \longrightarrow \mathbb{C P}^{1}$ be a degree $d$ covering of the Riemann sphere $\mathbb{C P}^{1}$ by a (connected) Riemann surface $\Sigma$. The degree $d$ of the covering is defined as the number of sheets above a generic point of $\mathbb{C P}^{1}$. A partition $\mu=\left(\mu_{1}, \mu_{2}, \ldots, \mu_{\ell(\mu)}\right)$ of $d$ is a set of positive integers $\mu_{i}$ such that

$$
d=\sum_{i=1}^{\ell(\mu)} \mu_{i}:=|\mu| \quad \text { and } \quad \mu_{1} \geq \mu_{2} \geq \ldots \geq \mu_{\ell(\mu)}>0 .
$$

Another notation for the partition is $\mu=\left(1^{m_{1}} 2^{m_{2}} \ldots\right)$ where $m_{i}$ is the number of parts equal to $i$, with $\ell(\mu)$ being the total number of parts of the partition $\mu$. Ramification points of a given $d$-fold covering are classified by partitions of $d$ which determine the type of monodromy (permutation of sheets) in moving around the point. The partition corresponding to a generic point (without ramification) is $\left(1^{d}\right)$. A ramification point is called simple if the corresponding partition is of the type $\left(1^{d-2} 2\right)$ that means a permutation of two sheets.

Let $\mu, \bar{\mu}$ be two partitions of $d$. The double Hurwitz numbers $H_{d, l}(\mu, \bar{\mu})$ [21] are defined as the properly weighted numbers of topologically non-equivalent coverings having ramification points at 0 and $\infty$ of the types $\mu$ and $\bar{\mu}$ respectively, $l \geq 0$ simple ramification points $P_{1}, \ldots, P_{l} \in \mathbb{C P}^{1}$ and unramified over all points other than $0, P_{1}, \ldots, P_{l}, \infty$. The genus $g$ of $\Sigma$ is determined by the ramification data $l, \mu, \bar{\mu}$ with the use of the RiemannHurwitz formula

$$
2 g-2=l-\ell(\mu)-\ell(\bar{\mu}) .
$$

Let $F^{(H)}(\beta, Q, \mathbf{t}, \overline{\mathbf{t}})$, where $\mathbf{t}=\left\{t_{1}, t_{2}, \ldots\right\}, \overline{\mathbf{t}}=\left\{\bar{t}_{1}, \bar{t}_{2}, \ldots\right\}$ are the times and $\beta, Q$ are parameters, be the generating function for the double Hurwitz numbers:

$$
F^{(H)}(\beta, Q, \mathbf{t}, \overline{\mathbf{t}})=\sum_{l \geq 0} \frac{\beta^{l}}{l !} \sum_{d \geq 1} Q^{d} \sum_{|\mu|=|\bar{\mu}|=d} H_{d, l}(\mu, \bar{\mu}) \prod_{i=1}^{\ell(\mu)} \mu_{i} t_{\mu_{i}} \prod_{i=1}^{\ell(\bar{\mu})} \bar{\mu}_{i} \bar{t}_{\bar{\mu}_{i}} .
$$

Here we employ the notation of [27] (the parameters $\beta, Q$ are related to our $R, r_{0}$ as $\left.\beta=1 / R, Q=r_{0}^{2}\right)$. The sum over $d$ combined with the sum over partitions such that 
$|\mu|=|\bar{\mu}|=d$ can be written as a sum over all partitions $\mu, \bar{\mu}$ with the convention that $H_{d, l}(\mu, \bar{\mu})=0$ unless $|\mu|=|\bar{\mu}|$. In [21] Okounkov has proved that the (dispersionfull) tau-function

$$
\tau_{n}(\mathbf{t}, \overline{\mathbf{t}})=e^{\frac{1}{12} \beta n(n+1)(2 n+1)} Q^{\frac{1}{2} n(n+1)} \exp \left(F^{(H)}\left(\beta, e^{\beta\left(n+\frac{1}{2}\right)} Q, \mathbf{t}, \overline{\mathbf{t}}\right)\right)
$$

solves the 2D Toda lattice hierarchy of Ueno and Takasaki.

In order to extract the contribution of genus $g$ surfaces the following trick is usually applied. Let us rescale $\left\{t_{k}\right\},\left\{\bar{t}_{k}\right\}$ and $\beta$ by introducing a new parameter $\hbar$ as $t_{k} \rightarrow t_{k} / \hbar, \beta \rightarrow \hbar \beta$ and consider the modified generating function $F^{(H)}(\hbar ; \beta, Q, \mathbf{t}, \overline{\mathbf{t}}):=$ $\hbar^{2} F^{(H)}(\hbar \beta, Q, \mathbf{t} / \hbar, \overline{\mathbf{t}} / \hbar)$, then the series (4.2) having regard to the Riemann-Hurwitz formula acquires the form of the topological expansion

$$
F^{(H)}(\hbar ; \beta, Q, \mathbf{t}, \overline{\mathbf{t}})=\sum_{g \geq 0} \hbar^{2 g} F_{g}^{(H)}(\beta, Q, \mathbf{t}, \overline{\mathbf{t}}),
$$

where

$$
F_{g}^{(H)}=\sum_{d \geq 1|\mu|=|\bar{\mu}|=d} \frac{Q^{d} \beta^{\ell(\mu)+\ell(\bar{\mu})+2 g-2}}{(\ell(\mu)+\ell(\bar{\mu})+2 g-2) !} H_{d, \ell(\mu)+\ell(\bar{\mu})+2 g-2}(\mu, \bar{\mu}) \prod_{i=1}^{\ell(\mu)} \mu_{i} t_{\mu_{i}} \prod_{i=1}^{\ell(\bar{\mu})} \bar{\mu}_{i} \bar{t}_{\bar{\mu}_{i}}
$$

counts the connected coverings of genus $g$. In particular,

$$
F_{0}^{(H)}=\sum_{d \geq 1} \sum_{|\mu|=|\bar{\mu}|=d} \frac{Q^{d} H_{d, \ell(\mu)+\ell(\bar{\mu})-2}(\mu, \bar{\mu})}{\beta^{2}(\ell(\mu)+\ell(\bar{\mu})-2) !} \prod_{i=1}^{\ell(\mu)}\left(\beta \mu_{i} t_{\mu_{i}}\right) \prod_{i=1}^{\ell(\bar{\mu})}\left(\beta \bar{\mu}_{i} \bar{t}_{\bar{\mu}_{i}}\right)
$$

is the generating function for the numbers of the ramified coverings $\mathbb{C P}^{1} \longrightarrow \mathbb{C P}^{1}$. At $\beta=0$ we have $H_{k, 0}((k),(k))=1 / k$ and

$$
\left.F_{0}^{(H)}\right|_{\beta=0}=\sum_{k \geq 1} k Q^{k} t_{k} \bar{t}_{k}
$$

The partial derivatives of the function $F_{0}^{(H)}$ with respect to $Q$ and $\beta$ (at constant $t_{i}$ ) are given by the formulas

$$
\begin{gathered}
Q \partial_{Q} F_{0}^{(H)}=\sum_{k \geq 1} k t_{k} \partial_{t_{k}} F_{0}^{(H)} \\
\partial_{\beta} F_{0}^{(H)}=\frac{1}{2} \sum_{k, l \geq 1}\left(k l t_{k} t_{l} \partial_{t_{k+l}} F_{0}^{(H)}+(k+l) t_{k+l} \partial_{t_{k}} F_{0}^{(H)} \partial_{t_{l}} F_{0}^{(H)}\right)
\end{gathered}
$$

The first one easily follows from the general structure of the series (4.5): taking into account that $\prod_{i=1}^{\ell(\mu)} t_{\mu_{i}}=\prod_{k \geq 1} t_{k}^{m_{k}}$ and $d=|\mu|=\sum_{k} k m_{k}$, we see that acting by the differential operators in the both sides of (4.7) to each monomial in the series, we get the same result. The second formula is a non-trivial combinatorial statement [34]. The right hand side comes from the dispersionless limit of the cut-and-join operator [34] discussed in [27].

Let $F_{0}\left(\beta, r_{0}, t_{0}, \mathbf{t}, \overline{\mathbf{t}}\right)$ be our LG tau-function given by (3.21) or (3.28), regarded as a function of the independent times $t_{k}$, including $t_{0}$, and the parameters $\beta, r_{0}$. We claim that the precise relation between $F_{0}$ and $F_{0}^{(H)}$ is as follows:

$$
F_{0}=\frac{\beta t_{0}^{3}}{6}+t_{0}^{2} \log r_{0}+F_{0}^{(H)}\left(\beta, r_{0}^{2} e^{\beta t_{0}}, \mathbf{t}, \overline{\mathbf{t}}\right)
$$


(In particular, $\left.F_{0}\right|_{t_{0}=0}=F_{0}^{(H)}\left(\beta, r_{0}^{2}, \mathbf{t}, \overline{\mathbf{t}}\right)$.) Indeed, the "initial conditions" at $\beta=0$ given by (3.34) and (4.6) match, so it is enough to show that the $\beta$-derivatives of the both sides coincide. This is easy to check using (3.32) and (4.7), (4.8). Equation (1.7) from the Introduction is obtained from (4.9) at $r_{0}=1$.

\section{Conclusion}

We have seen that conformal maps of plane domains and connected genus-0 ramified coverings of the sphere are governed by the same "master function", $F_{0}$, which is a special solution of the dispersionless Toda lattice hierarchy. Its arguments (commuting flows of the Toda hierarchy) are harmonic moments of the domain in the former case and formal variables necessary for constructing the generating function of Hurwits numbers in the latter. The double Hurwitz numbers $H_{d, l}(\mu, \bar{\mu})$ for the genus-zero coverings are basically the coefficients of the Taylor expansion of $F_{0}$ around the point $t_{k}=0$. This suggests that there should exist a direct connection between conformal maps and enumerative algebraic geometry of ramified coverings. One may also hope that this connection will be helpful for effectivization of the Riemann mapping theorem in the spirit of [35].

\section{Acknowledgments}

The author thanks A.Alexandrov, M.Mineev-Weinstein, A.Morozov, P.Wiegmann and especially S.Natanzon for discussions. This work was supported in part by RFBR grant 11-02-01220, by joint RFBR grants 12-02-91052-CNRS, 12-02-92108-JSPS, by grant NSh3349.2012.2 for support of leading scientific schools and by Ministry of Science and Education of Russian Federation under contract 8207.

\section{References}

[1] A comprehensive list of relevant papers published prior to 1998 can be found in: K. A. Gillow and S. D. Howison, A bibliography of free and moving boundary problems for Hele-Shaw and Stokes flow, http://www.maths.ox.ac.uk/ howison/Hele-Shaw/

[2] D. Bensimon, L.P. Kadanoff, S. Liang, B.I. Shraiman and C. Tang, Rev. Mod. Phys. 58 (1986) 977-999.

[3] P. Etingof and A. Varchenko, Why does the boundary of a round drop becomes a curve of order four, University Lecture Series, 3, American Mathematical Society, Providence, RI, 1992

[4] B. Gustafsson, A. Vasil'ev, Conformal and Potential Analysis in Hele-Shaw Cells, Birkhäuser Verlag, 2006.

[5] M. Mineev-Weinstein, P. Wiegmann and A. Zabrodin, Integrable structure of interface dynamics, Phys. Rev. Lett. 84 (2000) 5106-5109, arXiv:nlin.SI/0001007. 
[6] P. Wiegmann and A. Zabrodin, Conformal maps and integrable hierarchies, Commun. Math. Phys. 213 (2000) 523-538.

[7] I. Kostov, I. Krichever, M. Mineev-Weinstein, P. Wiegmann and A. Zabrodin, $\tau$ function for analytic curves, in: Random Matrix Models and Their Applications, Math. Sci. Res. Inst. Publ. vol. 40, Cambridge University Press, pp. 285-299, 2001, arXiv:hep-th/0005259.

[8] M. Mineev-Weinstein and A. Zabrodin, Whitham-Toda hierarchy in the Laplacian growth problem, Proceedings of the Workshop NEEDS-99 (Crete, Greece, June 1999), J. Nonlin. Math. Phys. 8 (2001) 212-218.

[9] A. Marshakov, P. Wiegmann and A. Zabrodin, Integrable Structure of the Dirichlet Boundary Problem in Two Dimensions, Commun. Math. Phys. 227 (2002) 131-153.

[10] A. Zabrodin, The dispersionless limit of the Hirota equations in some problems of complex analysis, Theor. Math. Phys. 129 (2001) 1511-1525 (Teor. Mat. Fiz. 129 (2001) 239-257), arXiv:math.CV/0104169.

[11] I. Krichever, M. Mineev-Weinstein, P. Wiegmann and A. Zabrodin, Laplacian growth and Whitham equations of soliton theory, Physica D 198 (2004) 1-28, arXiv:nlin.SI/0311005.

[12] R. Teodorescu, E. Bettelheim, O. Agam, A. Zabrodin and P. Wiegmann, Semiclassical evolution of the spectral curve in the normal random matrix ensemble as Whitham hierarchy, Nucl. Phys. B700 (2004) 521-532, arXiv:hep-th/0407017; Normal random matrix ensemble as a growth problem, Nucl. Phys. B704 (2005) 407-444, arXiv:hep-th/0401165.

[13] A. Zabrodin, Matrix models and growth processes: from viscous flows to the quantum Hall effect, in: "Applications of Random Matrices in Physics", pp. 261-318, Ed. E.Brezin et al, Springer, 2006, arXiv:hep-th/0412219.

[14] A. Zabrodin, Growth processes related to the dispersionless Lax equations, Physica D235 (2007) 101-108, arXiv:math-ph/0609023.

[15] I. Krichever, The $\tau$-function of the universal Whitham hierarchy, matrix models and topological field theories, Comm. Pure Appl. Math. 47 (1994) 437-475, arXiv:hep-th/9205110;

I. Krichever, The dispersionless Lax equations and topological minimal models, Commun. Math. Phys. 143 (1991) 415-429.

[16] K. Takasaki and T. Takebe, Integrable hierarchies and dispersionless limit, Rev. Math. Phys. 7 (1995) 743-808;

K. Takasaki and T. Takebe, SDiff(2) Toda equation - hierarchy, tau function and symmetries, Lett. Math. Phys. 23 (1991) 205-214.

[17] K. Ueno and K. Takasaki, Toda lattice hierarchy, Advanced Studies in Pure Math. 4 (1984) 1-95 
[18] M. Mineev-Weinstein, M. Putinar and R. Teodorescu, Random matrices in 2D, Laplacian growth and operator theory, J. Phys. A: Math. Theor. 41 (2008) 263001, arXiv:0805.0049.

[19] S. Lando, Ramified coverings of the two-dimensional sphere and intersection theory in spaces of meromorphic functions on algebraic curves, Russ. Math. Surv. 57:3 (2002) 463533.

[20] R. Pandharipande, The Toda equations and the Gromov-Witten theory on the Riemann sphere, Lett. Math. Phys. 53 (2000) 59-74, arXiv:math.AG/9912166.

[21] A. Okounkov, Toda equations for Hurwitz numbers, Math. Res. Lett. 7 (2000) 447453.

[22] A. Okounkov and R. Pandharipande, Gromov-Witten theory, Hurwitz theory, and completed cycles , Annals of Mathematics, 163 (2006) 517-560, arXiv:math/0204305.

[23] M. Kazarian and S. Lando, An algebro-geometric proof of Witten's conjecture, J. Amer. Math. Soc. 20 (2007) 1079-1089.

[24] G. Borot, B. Eynard, M. Mulase and B. Safnuk, A matrix model for simple Hurwitz numbers, and topological recursion, J. Geom. Phys. 61 (2011) 522-540.

[25] A. Alexandrov, Matrix models for random partitions, Nucl. Phys. B851 (2011) 620650, arXiv:1005.5715.

[26] A. Alexandrov, A. Mironov, A. Morozov and S. Natanzon, Integrability of Hurwitz partition functions. I. Summary, J. Phys. A: Math. Theor. 45 (2012) 045209, arXiv:1103.4100;

A. Mironov, A. Morozov and S. Natanzon, Integrability properties of Hurwitz partition functions. II. Multiplication of cut-and-join operators and WDVV equations, JHEP 11 (2011) 097.

[27] K. Takasaki, Generalized string equations for double Hurwitz numbers, J. Geom. Phys. 62 (2012), 1135-1156.

[28] S. Howison, Complex variable method in Hele-Show moving boundary problems, Euro. J. of Appl. Math. 3 (1992) 209-224.

[29] P.Ya. Polubarinova-Kochina, On the motion of the oil contour, Dokl. Akad. Nauk. S.S.S.R. 47 254-257 (in Russian);

L.A. Galin, Unsteady filtration with a free surface, Dokl. Akad. Nauk S.S.S.R. (1945) 47 246-249 (in Russian).

[30] S. Richardson, Hele-Shaw flows with a free boundary produced by injection of fluid into a narrow channel, J. Fluid Mech. 56 (1972) 609-618.

[31] B.A. Dubrovin and S.P. Novikov, Hamiltonian formalism of one-dimensional systems of the hydrodynamic type and the Bogolyubov-Whitham averaging method, Soviet Math. Dokl. 27 (1983) 665-669;

S.P. Tsarev, Poisson brackets and one-dimensional Hamiltonian systems of hydrodynamic type, Soviet Math. Dokl. 31 (1985) 488-491. 
[32] A. Orlov and E. Shulman, Additional symmetries for integrable equations and conformal algebra representation, Lett. Math. Phys. 12 (1986) 171-179.

[33] P.J. Davis, The Schwarz function and its applications, The Carus Math. Monographs, No. 17, The Math. Assotiation of America, Buffalo, N.Y., 1974

[34] I.P. Goulden and D.M. Jackson, Transitive factorizations into transpositions and holomorphic mappings on the sphere, Proc. Amer. Math. Soc. 125 (1997) 51-60.

[35] S. Natanzon, Integrable systems and effectivization of the Riemann theorem about domains of the complex plane, Moscow Math. J. 3 (2003) 541-549;

S. Natanzon, Towards an effectivisation of the Riemann theorem, Ann. Global Anal. Geom. 28 (2005) 233-255;

Yu. Klimov, A. Korzh and S. Natanzon, From 2D Toda hierarchy to conformal maps for domains of Riemann sphere, Amer. Math. Soc. Trans. (2) 212 (2004) 207-218. 